Provided for non-commercial research and education use. Not for reproduction, distribution or commercial use.

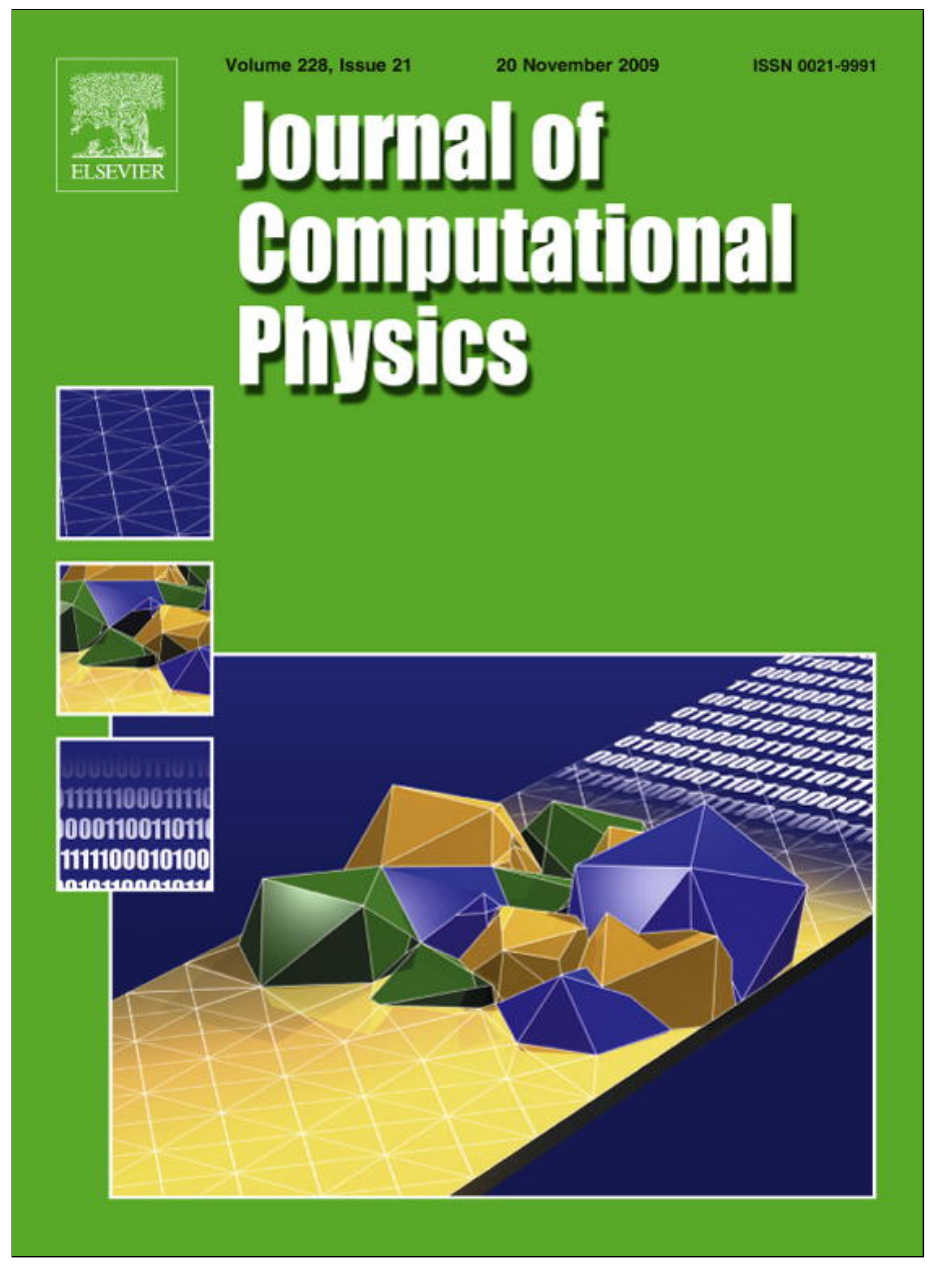

This article appeared in a journal published by Elsevier. The attached copy is furnished to the author for internal non-commercial research and education use, including for instruction at the authors institution and sharing with colleagues.

Other uses, including reproduction and distribution, or selling or licensing copies, or posting to personal, institutional or third party websites are prohibited.

In most cases authors are permitted to post their version of the article (e.g. in Word or Tex form) to their personal website or institutional repository. Authors requiring further information regarding Elsevier's archiving and manuscript policies are encouraged to visit:

http://www.elsevier.com/copyright 


\title{
An accurate, stable and efficient domain-type meshless method for the solution of MHD flow problems
}

\author{
G.C. Bourantas a, E.D. Skouras ${ }^{\text {b,c }}$, V.C. Loukopoulos ${ }^{\text {d,* }}$, G.C. Nikiforidis ${ }^{a}$ \\ ${ }^{a}$ Department of Medical Physics, School of Medicine, University of Patras, GR 26500, Rion, Greece \\ ${ }^{\mathrm{b}}$ Department of Chemical Engineering, University of Patras, GR 26500, Rion, Greece \\ ${ }^{\mathrm{c}}$ Institute of Chemical Engineering and High Temperature Chemical Processes, Foundation for Research and Technology, P.O. Box 1414, GR-26504, Patras, Greece \\ ${ }^{\mathrm{d}}$ Department of Physics, University of Patras, Patras, GR 26500, Rion, Greece
}

\section{A R T I C L E I N F O}

\section{Article history:}

Received 24 December 2008

Received in revised form 14 June 2009

Accepted 28 July 2009

Available online 5 August 2009

\section{PACS:}

47.65. $-\mathrm{d}$

47.11. $-\mathrm{j}$

$52.30 \mathrm{Cv}$

02.60. $-\mathrm{X}$

Keywords:

Meshless

Point collocation

MLS

MHD

Hartmann number

\begin{abstract}
A B S T R A C T
The aim of the present paper is the development of an efficient numerical algorithm for the solution of magnetohydrodynamics flow problems for regular and irregular geometries subject to Dirichlet, Neumann and Robin boundary conditions. Toward this, the meshless point collocation method (MPCM) is used for MHD flow problems in channels with fully insulating or partially insulating and partially conducting walls, having rectangular, circular, elliptical or even arbitrary cross sections. MPC is a truly meshless and computationally efficient method. The maximum principle for the discrete harmonic operator in the meshfree point collocation method has been proven very recently, and the convergence proof for the numerical solution of the Poisson problem with Dirichlet boundary conditions have been attained also. Additionally, in the present work convergence is attained for Neumann and Robin boundary conditions, accordingly. The shape functions are constructed using the Moving Least Squares (MLS) approximation. The refinement procedure with meshless methods is obtained with an easily handled and fully automated manner. We present results for Hartmann number up to $10^{5}$. The numerical evidences of the proposed meshless method demonstrate the accuracy of the solutions after comparing with the exact solution and the conventional FEM and BEM, for the Dirichlet, Neumann and Robin boundary conditions of interior problems with simple or complex boundaries.
\end{abstract}

(c) 2009 Elsevier Inc. All rights reserved.

\section{Introduction}

Poisson, Helmholtz, and diffusion-convection equations are fundamental modeling components of the behavior of several physical phenomena and industrial processes. In order to solve these physical problems, governed by partial differential equations, researchers and scientists have proposed various approximations. One generally accepted route for obtaining numerical solutions to these partial differential equations is the application of the finite element method (FEM). Although FEM has a key advantage over other numerical methods in that it can handle arbitrary problem geometries, it usually requires a body-fitted mesh. FEM users usually have to write their own mesh generation programs, a process far more difficult and time-consuming than the solvers of the FEM programs [1], due to the shortage of a universally accepted mesh generation program that is efficient, freely available, and capable of generating 2D, 3D, and 4D (time-varying) meshes. In order to avoid body-fitted mesh generation, a meshless method such as the meshless point collocation method (MPCM) may be used alternatively.

\footnotetext{
* Corresponding author. Tel.: +30 2610 997447; fax: +30 2610996089.

E-mail addresses: bourantas@med.upatras.gr (G.C. Bourantas), eugene@iceht.forth.gr (E.D. Skouras), vxloukop@physics.upatras.gr (V.C. Loukopoulos), gnikif@med.upatras.gr (G.C. Nikiforidis).
} 
Meshless methods provide a viable alternative to grid-based flow computation since they do not require conventional grid structures, alleviating many issues related to grid generation. Instead of relying on stencils, elements, or control volumes, meshless methods make use of point clouds to discretize the mathematical equations governing incompressible flow. The meshless numerical method has become an attractive alternative to the finite element (FEM) and the boundary element method (BEM) due to its inherent advantage of avoiding meshing and remeshing, the efficient treatment of complicated load conditions and, thus, avoiding mesh distortion in large deformation problems. Furthermore, the refinement procedure with meshless methods is obtained with an easily handled and fully automated manner.

The meshless method is usually divided into two main categories: the boundary-type meshless method, and the domaintype meshless method. Herein, the latter procedure is adopted. Recent works have indicated that highly accurate results may be obtained with meshless methods, as compared to grid-based methods [1,2]. Over the last years, several meshless methods have been proposed, as the Smoothed Particle Hydrodynamics (SPH) [3], the Diffuse Element Method (DEM) [4], the Element Free Galerkin method (EFG) [5], the Reproducing Kernel Particle Method (RKPM) [6,7], the Partition of Unity Finite Element method (PUFEM) [8], the h-p Clouds [9], the Moving Least-Square Reproducing Kernel method (MLSRK) [10], the meshless Local Boundary Integral Equation method (LBIE) [11], the Meshless Local Petrov-Galerkin method (MLPG) [12], meshless point collocation methods using reproducing kernel approximations [13], the Merhod of Fundamental Solutions (MFS) [14], the Method of Particular Solutions (MPS) [15] and more. In the present work we imply the MPC method for the solution of equations that describe the MHD flow. The construction of approximation functions can be performed entirely in terms of point locations using the Moving Least Squares (MLS) method. The discretization of the domain of interest is accomplished using a set of scattered points, and the shape functions are established at the global level without the requirement of any mesh.

Incompressible magnetohydrodynamics (MHD) describes the flow of a viscous, incompressible and electrically conducting fluid. The governing partial differential equations are obtained by coupling the incompressible Navier-Stokes equations with Maxwell's equations. The aforementioned equations arise in several engineering applications, such as, for example, liquid metals in magnetic pumps or aluminum electrolysis. The magnetohydrodynamic flow problems through ducts are frequently encountered in nuclear reactors and magnetohydrodynamic generators, as well as in pumps and accelerators. Also, direct links of magnetohydrodynamic with medicine comprise blood flow measurements and magnetic occlusion of arterial aneurysms [16-20].

Due to the coupling of the equations of fluid mechanics and electrodynamics, exact solutions are available solely for some simple geometries under very simple boundary conditions [19]. Therefore, several numerical techniques, such as the finite

Case 1

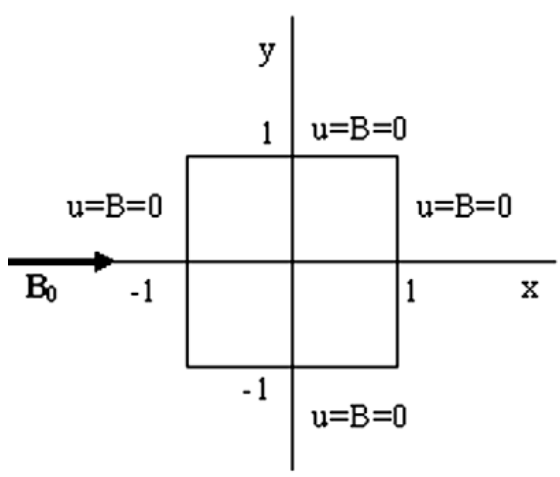

Case 3

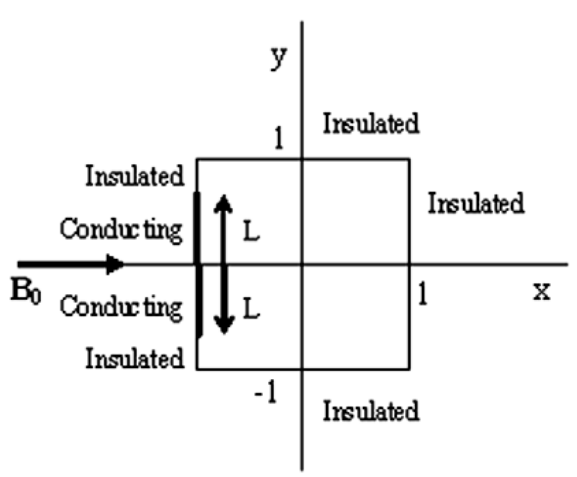

Case 2

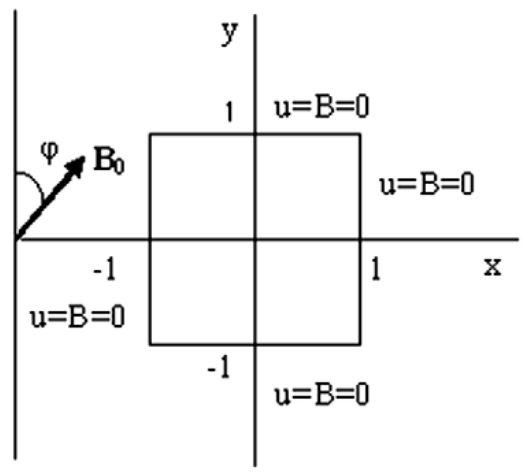

Case 4

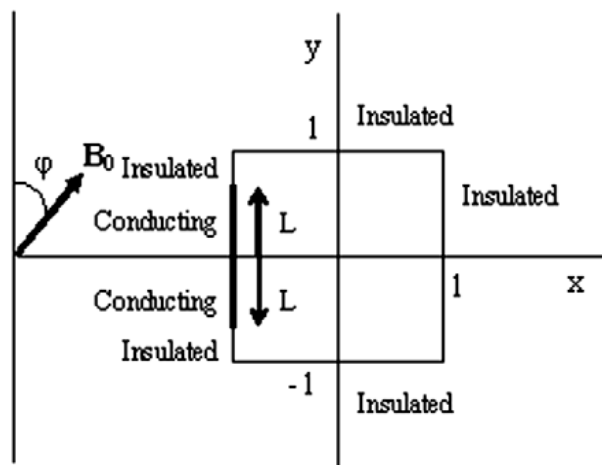

Fig. 1. Square channel flow with external applied magnetic field. 
difference method (FDM) [20], the finite element method (FEM) [21-23], the boundary element method (BEM) [18], and the fundamental solution method [19] have been used to obtain approximate solutions for MHD flow problems. In most cases, results were obtained for small $(<100)$ and moderate $(>100$ and $<1000)$ Hartmann numbers, while in [22] results were obtained for Hartmann numbers up to $10^{5}$. Nevertheless, various types of difficulties are referred in regard to very fine meshes for large values of Hartmann number, which is computationally very expensive, memory and time-consuming.

In recent years, research on meshless (meshfree) methods has made significant progress in science and engineering, particularly in the area of computational mechanics. In the present paper, our aim is to establish the MPCM solution of MHD duct flow equations for large values of Hartman number in the original coupled form, which are of the convection-diffusion type. The present work transforms the MHD differential equations into the discretized matrix-vector form $\boldsymbol{L} \boldsymbol{u}=\boldsymbol{f}$, where the matrix operator $\boldsymbol{L}$ contains the derivative operators appearing in the MHD flow equations, and solves the resulting sparse discretized equations directly. The meshless point collocation method is used for MHD flow problem in channels with insulating walls or partial insulating and partial conducting walls, having rectangular, circular, elliptical or arbitrary cross sections. Despite the numerical efficiency and the implementation benefits of the meshless point collocation method, the maximum principle for the discrete harmonic operator [24], and the convergence proof for the numerical solution of the Poisson problem with Dirichlet boundary conditions have been attained for this method just recently. Additionally, in the
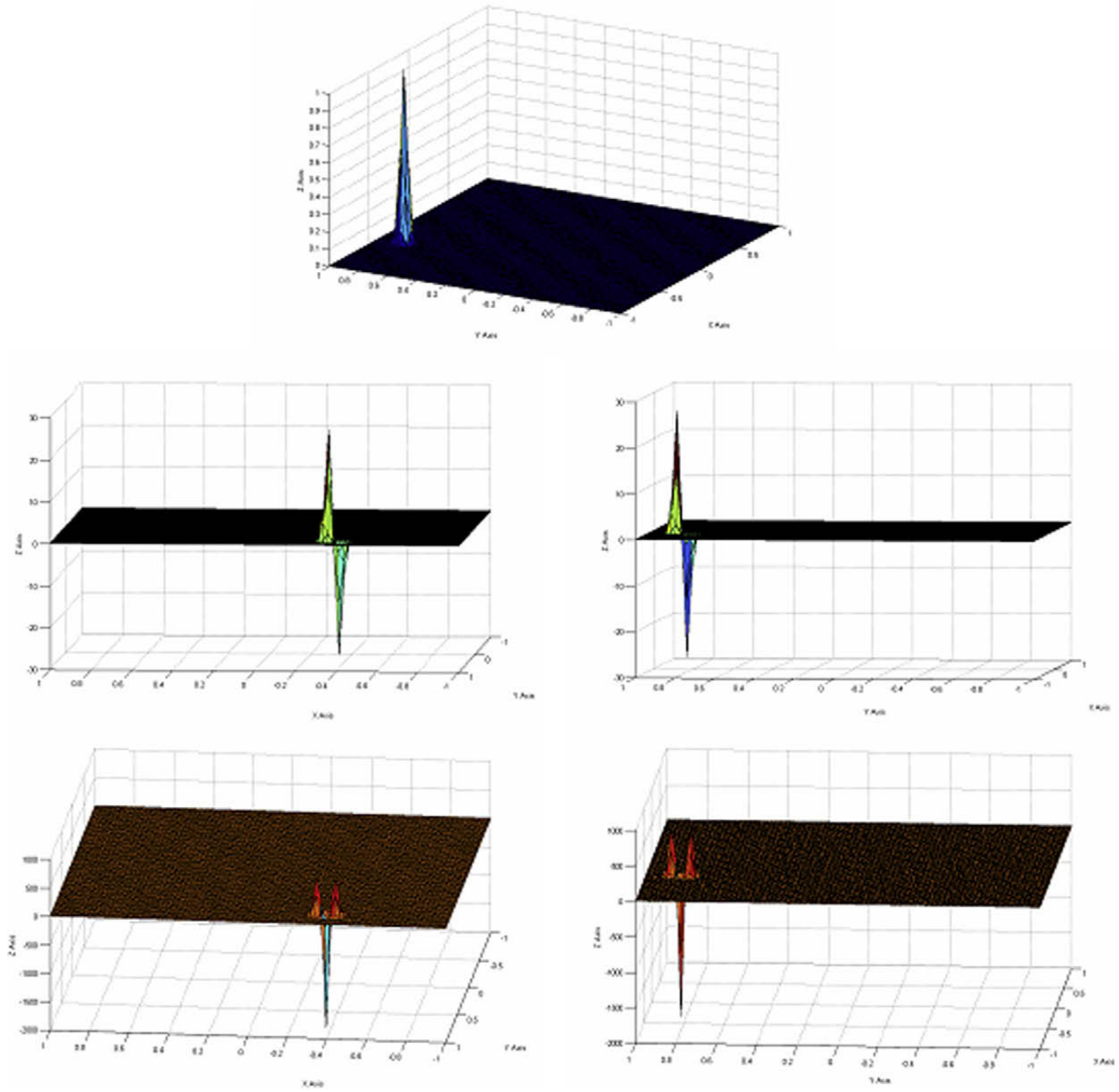

Fig. 2. The Gaussian weight functions: (a) $W_{I}^{[(0,0)]}$, (b) $W_{I}^{[(1,0)]}$, (c) $W_{I}^{[(0,1)]}$, (d) $W_{I}^{[(2,0)]}$, and (e) $W_{I}^{[(0,2)]}$. 
present work convergence is attained for Neumann and Robin boundary conditions, accordingly, using suitable node distributions [24]. To this end, a smart local refinement procedure for additional nodes insertion at each site, in relation to an error indicator is used here for the improvement of the numerical accuracy with respect to these convergence conditions. Four cases with varying magnetic field and boundary conditions on the walls for a rectangular duct are considered in the present work. Results for regular geometries and large values of Hartmann number (up to $10^{5}$ ), as well as for irregular geometries, are presented.

\section{Problem formulation}

A steady, laminar, fully developed flow of a viscous, incompressible and electrically conducting fluid in a straight thinwalled duct is assumed. The fluid is subject to a constant and uniformly applied magnetic field, imposed in the $x$-direction with a constant axial pressure gradient, $-\kappa$. The equations governing the steady flow are [23]:

$$
\begin{aligned}
& \mathbf{v} \cdot \nabla \mathbf{v}=-\frac{1}{\rho} \nabla p+v \nabla^{2} \mathbf{v}+\frac{1}{\rho} \mathbf{j} \times \mathbf{B}, \\
& \nabla \cdot \mathbf{v}=0, \\
& \mathbf{j}=\nabla \times \mathbf{H}=\sigma(-\nabla F+\mathbf{v} \times \mathbf{B}), \\
& \nabla \cdot \mathbf{B}=0, \quad \mathbf{B}=\mu \mathbf{H},
\end{aligned}
$$

where $\mathbf{v}$ is the velocity, $p$ is the pressure, $v$ is the kinetic viscosity, $\rho$ is the density, $\mathbf{j}$ is the current, B is the magnetic induction, $\mathbf{H}$ is the magnetic field, $F$ is the electric potential, $\sigma$ is the conductivity of the fluid, and $\mu$ is the permeability.

For the case where (i) $\mathbf{v}=(0,0, u), \mathbf{B}=\left(\mu H_{0}, 0, B\right)$, (ii) there is no variation in the $z$-direction except for the pressure gradient $-\kappa$, and (iii) the duct cross-section has a typical dimension a, the equations may be written in non-dimensional form as:

$$
\begin{aligned}
& \nabla^{2} u+M \frac{\partial B}{\partial x}=-1, \\
& \nabla^{2} B+M \frac{\partial u}{\partial x}=0, \quad \text { in } \Omega .
\end{aligned}
$$

The non-dimensionalization of Eqs. (2.5) and (2.6) was performed with the aid of suitable non-dimensional variables [23]. The Hartmann number $M$ appearing in (2.5) and (2.6) is given by $M=a B_{0} \sqrt{\sigma / \rho v}$, where $\mathbf{B}_{0}$ is the intensity of the applied magnetic field. The velocity on the walls, $\partial \Omega$, satisfies the no-slip boundary condition $u=0$, while $B=0$ on $\partial \Omega$ ensures that the walls of the duct are insulating.

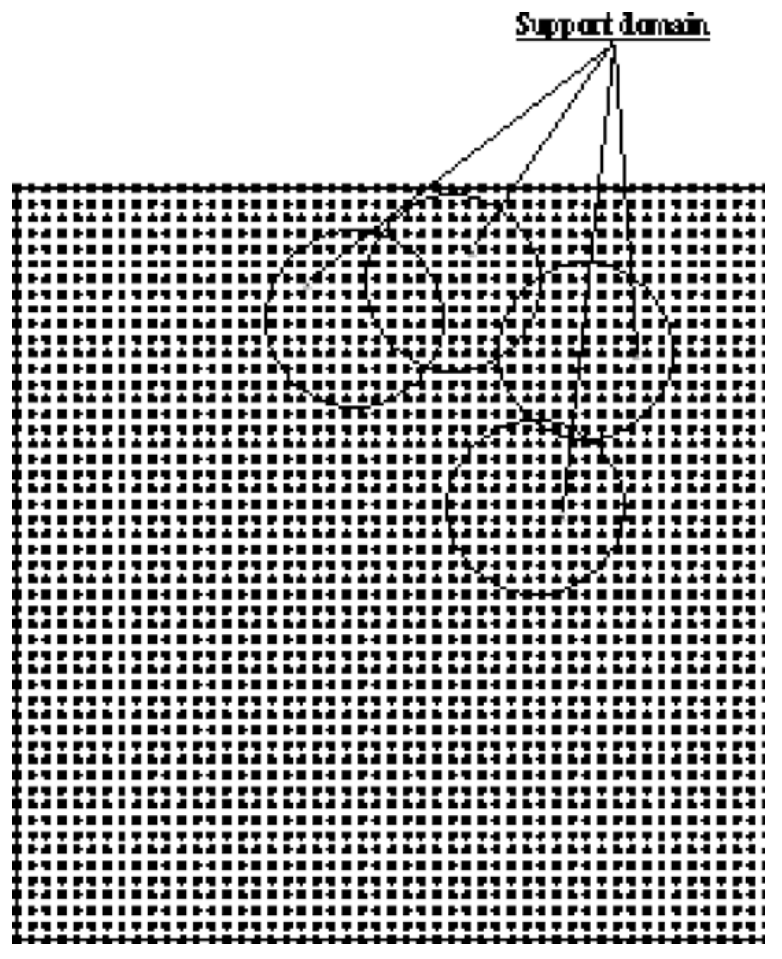

Fig. 3. Support domain. 
Table 1

The flow field and the magnetic field at $M=100$.

\begin{tabular}{|c|c|c|c|c|c|c|c|}
\hline$x$ & $y$ & $U_{h}(\mathrm{FEM})$ & $U_{s}(\mathrm{MPCM})$ & $U_{\text {best }}$ & $B_{h}(\mathrm{FEM})$ & $B_{h}(\mathrm{MPCM})$ & $B_{\text {best }}$ \\
\hline 0.00 & 0.00 & 0.0100000 & 0.0099998 & 0.0100000 & 0.0000000 & 0.0000001 & 0.0000000 \\
\hline 0.25 & 0.00 & 0.0100000 & 0.0099998 & 0.0100000 & -0.0025000 & -0.0024999 & -0.0025000 \\
\hline 0.50 & 0.00 & 0.0100000 & 0.0099998 & 0.0100000 & -0.0050000 & -0.0049999 & -0.0050000 \\
\hline 0.75 & 0.00 & 0.0100000 & 0.0100000 & 0.0100000 & -0.0075000 & -0.0075001 & -0.0075000 \\
\hline 0.00 & 0.25 & 0.0100000 & 0.0100000 & 0.0100000 & 0.0000000 & 0.0000000 & 0.0000000 \\
\hline 0.25 & 0.25 & 0.0100000 & 0.0099999 & 0.0100000 & -0.0025000 & -0.0024999 & -0.0025000 \\
\hline 0.50 & 0.25 & 0.0099999 & 0.0099999 & 0.0100000 & -0.0050000 & -0.0049998 & -0.0050000 \\
\hline 0.00 & 0.50 & 0.0099993 & 0.0099992 & 0.0099992 & 0.0000000 & 0.0000002 & -0.0000000 \\
\hline 0.25 & 0.50 & 0.0099983 & 0.0099982 & 0.0099981 & -0.0024984 & -0.0024977 & -0.0024982 \\
\hline 0.50 & 0.50 & 0.0099947 & 0.0097651 & 0.0099944 & -0.0049947 & -0.0049944 & -0.0049944 \\
\hline 0.75 & 0.50 & 0.0099873 & 0.0097187 & 0.0099868 & -0.0074873 & -0.0074877 & -0.0074868 \\
\hline 0.00 & 0.75 & 0.0097662 & 0.0095878 & 0.0097614 & 0.0000000 & -0.0000010 & 0.0000000 \\
\hline 0.25 & 0.75 & 0.0097209 & 0.0099947 & 0.0097163 & -0.0023043 & -0.0023045 & -0.0023030 \\
\hline 0.50 & 0.75 & 0.0095898 & 0.0099879 & 0.0095858 & -0.0046050 & -0.0046042 & -0.0046024 \\
\hline 0.75 & 0.75 & 0.0093899 & 0.0093882 & 0.0093863 & -0.0068903 & -0.0068914 & -0.0068869 \\
\hline
\end{tabular}

Table 2

The flow field and the magnetic field at $M=500$.

\begin{tabular}{|c|c|c|c|c|c|c|c|}
\hline$x$ & $y$ & $U_{h}(\mathrm{FEM})$ & $U_{s}(\mathrm{MPCM})$ & $U_{\text {best }}$ & $B_{h}(\mathrm{FEM})$ & $B_{h}(\mathrm{MPCM})$ & $B_{\text {best }}$ \\
\hline 0.00 & 0.00 & 0.0020000 & 0.0019999 & 0.0020000 & 0.0000000 & 0.0000000 & 0.0000000 \\
\hline 0.25 & 0.00 & 0.0020000 & 0.0019999 & 0.0020000 & -0.0005000 & -0.0004999 & -0.0005000 \\
\hline 0.50 & 0.00 & 0.0020000 & 0.0019999 & 0.0020000 & -0.0010000 & -0.0009999 & -0.0010000 \\
\hline 0.75 & 0.00 & 0.0020000 & 0.0020000 & 0.0020000 & -0.0015000 & -0.0015000 & -0.0015000 \\
\hline 0.00 & 0.25 & 0.0020000 & 0.0020000 & 0.0020000 & 0.0000000 & -0.0000000 & 0.0000000 \\
\hline 0.25 & 0.25 & 0.0020000 & 0.0020000 & 0.0020000 & -0.0005000 & -0.0005000 & -0.0005000 \\
\hline 0.50 & 0.25 & 0.0020000 & 0.0020000 & 0.0020000 & -0.0010000 & -0.0010000 & -0.0010000 \\
\hline 0.75 & 0.25 & 0.0020000 & 0.0019999 & 0.0020000 & -0.0015000 & -0.00150000 & -0.0015000 \\
\hline 0.00 & 0.50 & 0.0020000 & 0.0019999 & 0.0020000 & -0.0000000 & 0.0000000 & 0.0000000 \\
\hline 0.25 & 0.50 & 0.0020000 & 0.0019999 & 0.0020000 & -0.0005000 & -0.0004999 & -0.0005000 \\
\hline 0.50 & 0.50 & 0.0020000 & 0.0019999 & 0.0020000 & -0.0010000 & -0.0010000 & -0.0010000 \\
\hline 0.75 & 0.50 & 0.0020000 & 0.0019999 & 0.0020000 & -0.0015000 & -0.0015000 & -0.0015000 \\
\hline 0.00 & 0.75 & 0.0020000 & 0.0020001 & 0.0020000 & 0.0000000 & -0.0000001 & 0.0000000 \\
\hline 0.25 & 0.75 & 0.0019999 & 0.0020000 & 0.0019999 & -0.0005000 & -0.0005001 & -0.0004999 \\
\hline 0.50 & 0.75 & 0.0019998 & 0.0019998 & 0.0019997 & -0.000998 & -0.0009998 & -0.0009997 \\
\hline 0.75 & 0.75 & 0.0019994 & 0.0019991 & 0.0019992 & -0.0014994 & -0.0014993 & -0.0014992 \\
\hline
\end{tabular}
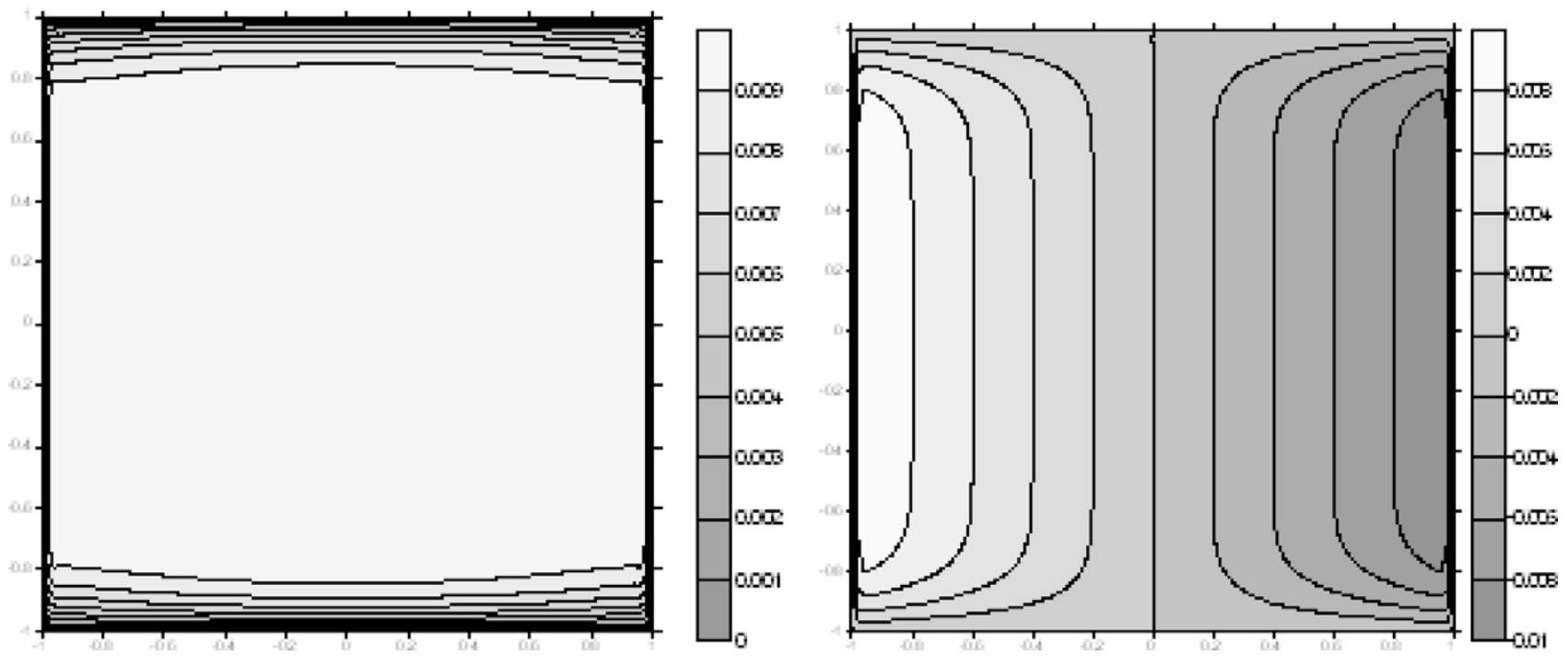

Fig. 4. Equivelocity lines and induced magnetic field lines for $M=100$ and $\phi=\frac{\pi}{2}$ in rectangular duct without local refinement, $(u$, max $=0.0100000$, min $=0$ and $B, \max =0.0095287, \min =-0.0095287)$. 
For the case where a constant and uniform oblique magnetic field is applied, the coupled system of equations in the velocity and magnetic field can be put in the following non-dimensional form [19]

$$
\begin{aligned}
& \nabla^{2} u+M_{x} \frac{\partial B}{\partial x}+M_{y} \frac{\partial B}{\partial y}=-1, \\
& \nabla^{2} B+M_{x} \frac{\partial u}{\partial x}+M_{y} \frac{\partial u}{\partial y}=0, \quad \text { in } \Omega .
\end{aligned}
$$

Hartmann number $M$ is the norm of the vector $\mathbf{M}=\left(M_{x}, M_{y}\right)$. The fluid is driven down the duct by means of a constant pressure gradient. The applied magnetic field of intensity $\mathbf{B}_{0}$ acts in a direction lying in the $x y$-plane but forming an angle $\phi$ with $y$-axis. The components of the vector $\mathbf{M}$ take the form

$$
\begin{aligned}
& M_{x}=M \sin \varphi, \\
& M_{y}=M \cos \varphi,
\end{aligned}
$$

In general, the boundary conditions can be expressed as

$$
\begin{array}{llll}
u=u_{p} & \text { on } & \partial \Omega & \text { (Dirichlet (essential) boundary conditions), } \\
B=B_{p} & \text { on } & \Gamma_{u} & \text { (Dirichlet boundary conditions), }
\end{array}
$$
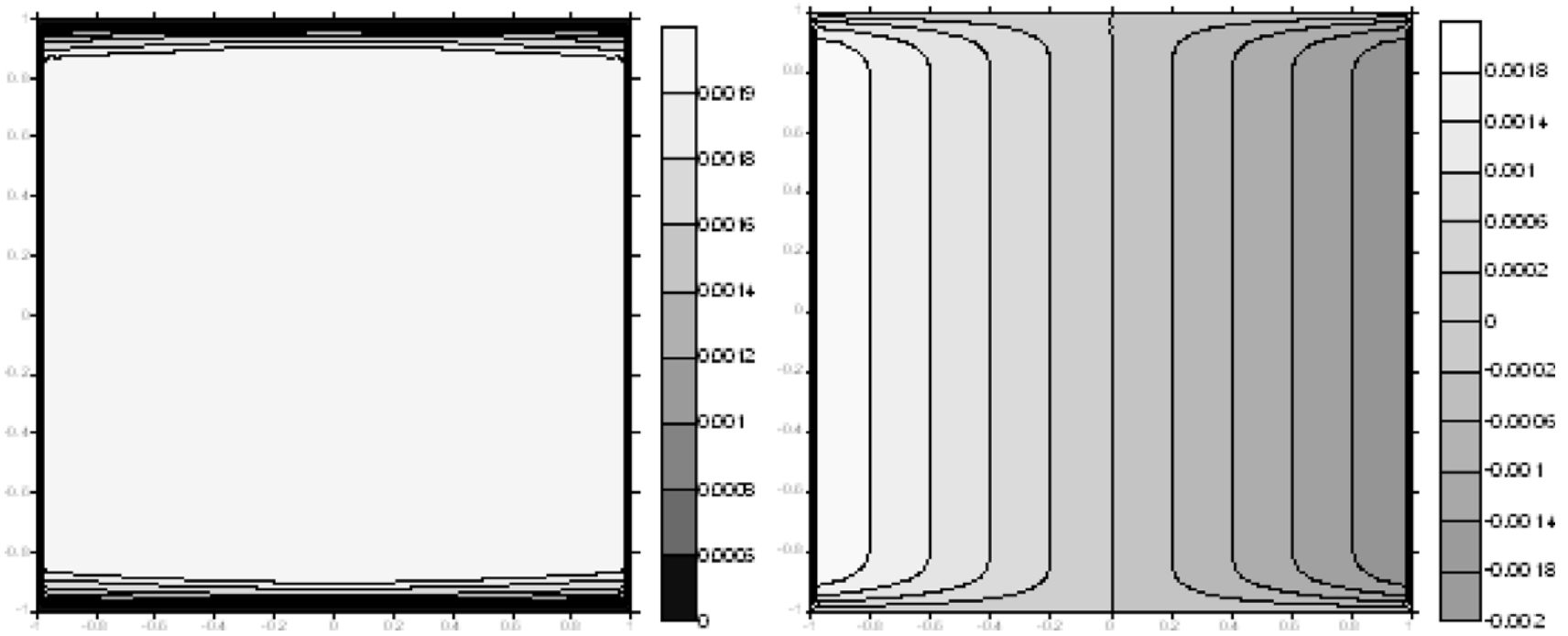

Fig. 5. Equivelocity lines and induced magnetic field lines for $M=500$ and $\phi=\frac{\pi}{2}$ in rectangular duct without local refinement, $(u$, $\max =0.0020010$, min $=0$ and $B$, $\max =0.00192287$, $\min =-0.00192287)$.
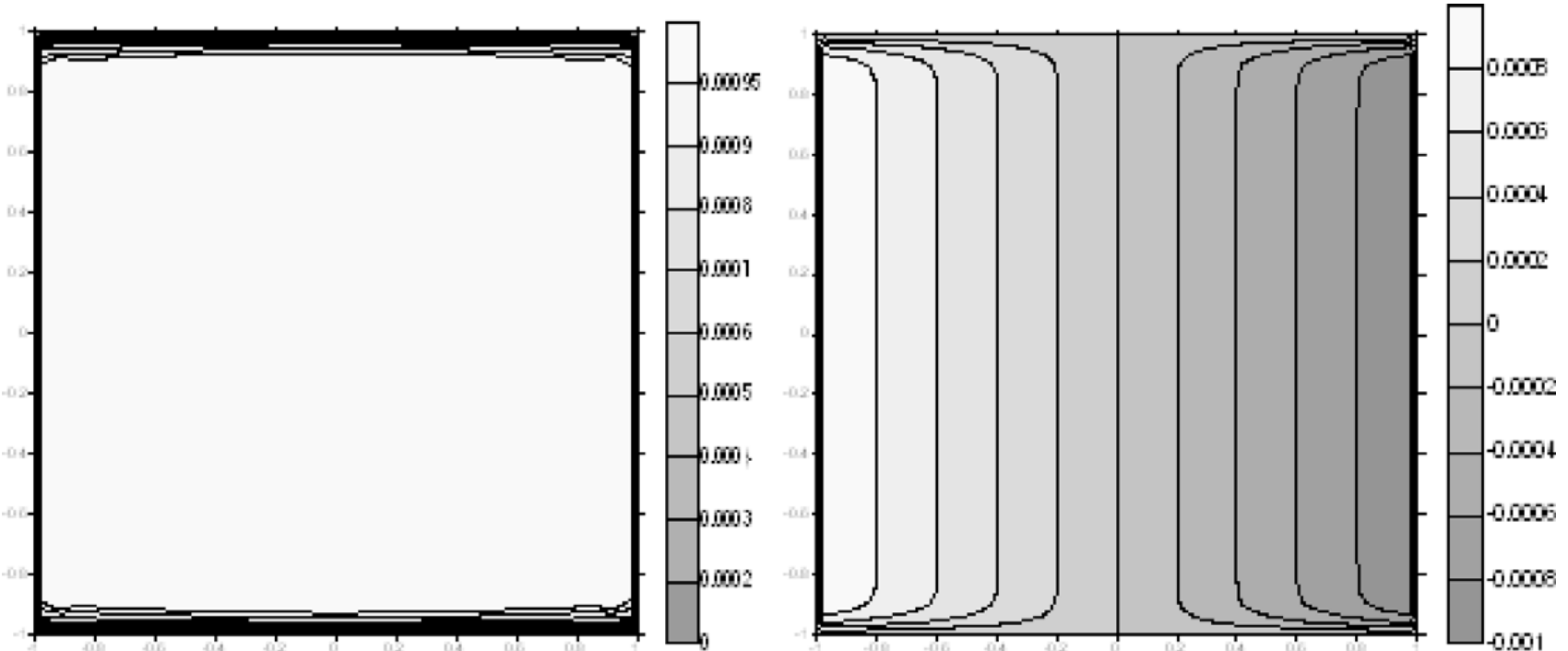

Fig. 6. Equivelocity lines and induced magnetic field lines for $M=1000$ and $\phi=\frac{\pi}{2}$ in rectangular duct without local refinement, $(u$, max $=0.0010001$, min $=0$ and $B, \max =0.000950087, \min =-0.000950087)$. 
$\frac{\partial B}{\partial \mathbf{n}}=t \quad$ on $\quad \Gamma_{t} \quad$ (Neumann (natural) boundary conditions)

where $\partial \Omega=\Gamma_{u} \cup \Gamma_{t}$ is the boundary of $\Omega$ with $\Gamma_{u} \cap \Gamma_{t}=\emptyset . \Gamma_{u}$ and $\Gamma_{t}$ are the insulating and conducting parts of the boundary $\partial \Omega$, respectively. $\mathbf{n}$ is the vector of unit outward normal at a point on the natural boundary.

In total, we consider four cases: a rectangular duct with insulating walls (Case 1); a rectangular duct with insulating walls, under the influence of an oblique magnetic field (Case 2); a rectangular duct with partly insulating, partly conducting walls (Case 3); and a rectangular duct with partly insulating, partly conducting walls, under the influence of an oblique magnetic field (Case 4). All these cases are presented in Fig. 1.

\section{Numerical method}

\subsection{Moving least squares approximation}

Let $u(\boldsymbol{x})$ be the unknown function of the field variable defined in the domain $\Omega$. The function $u^{h}(\boldsymbol{x})$ is the approximation of function $u(\boldsymbol{x})$ at point $\boldsymbol{x}$. The field function is defined using the Moving Least Squares (MLS) approximation as
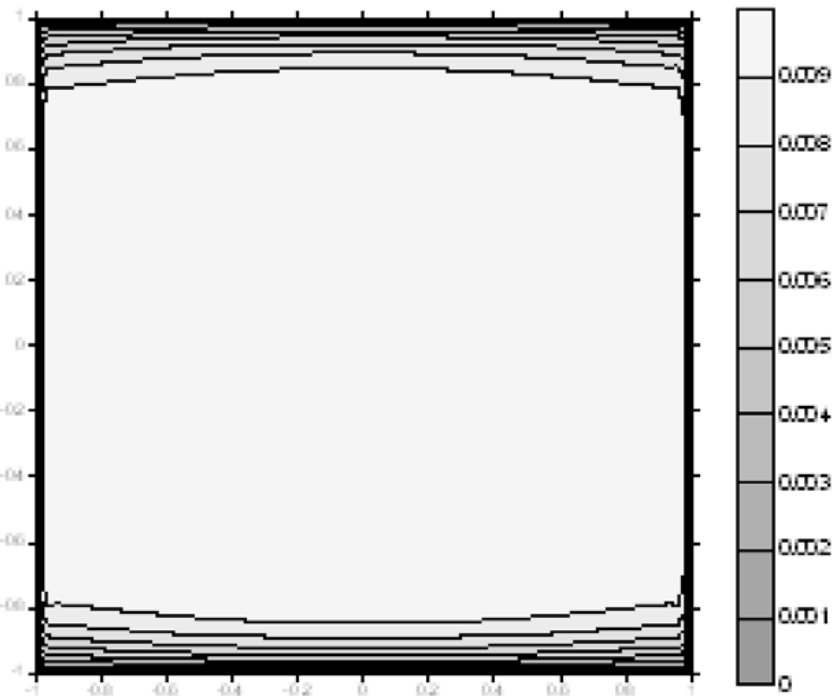

(u, $\max =0.0100000$, mitr $=0)$
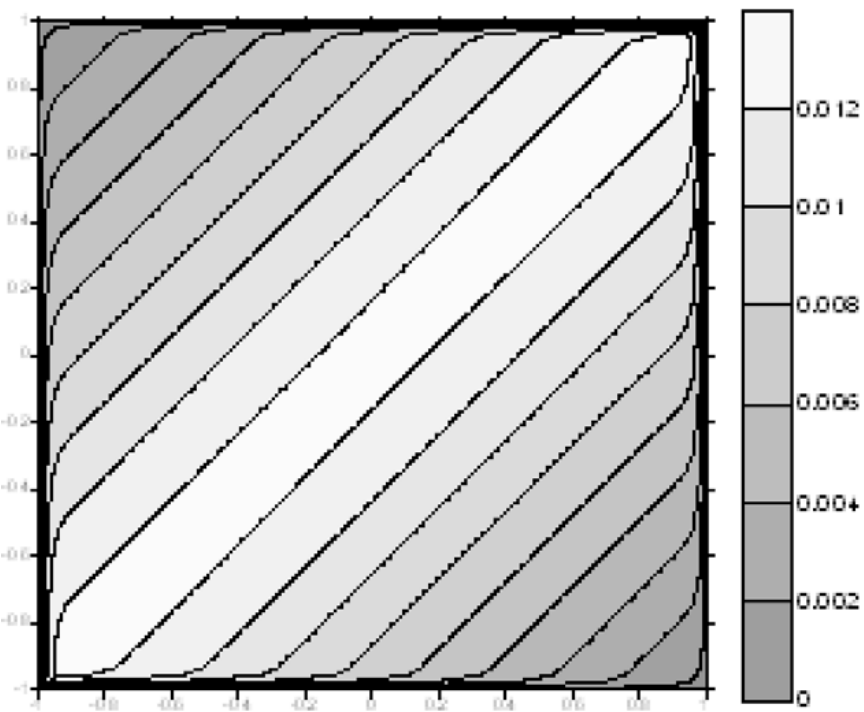

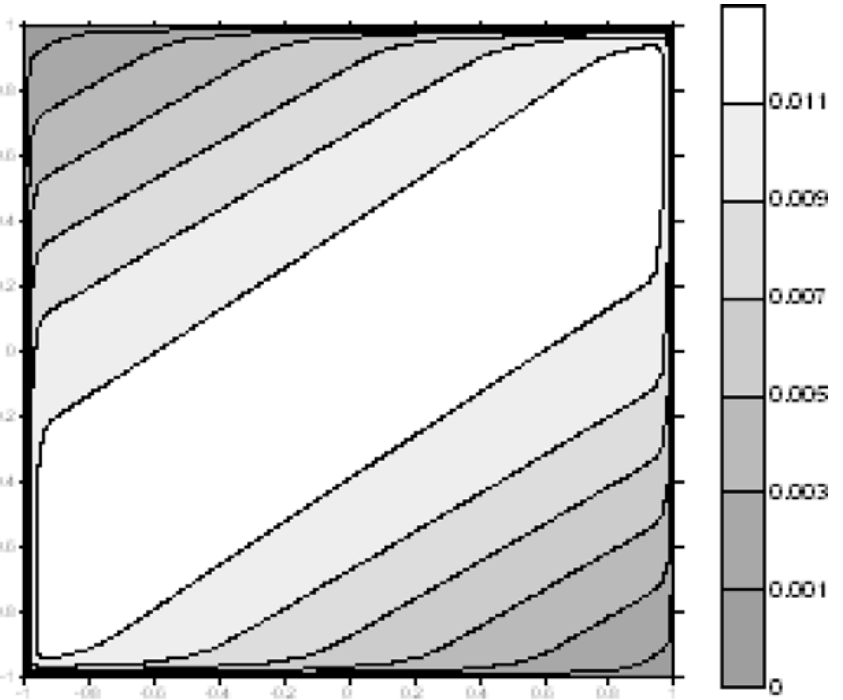

$(u, \max =0.0115389$, thitr $=0)$

\section{$(u, \operatorname{tn} a x=0.0130108, \operatorname{tnitr}=0)$}

Fig. 7. Equivelocity lines for $M=100$ and $\phi=\frac{\pi}{2}, \frac{\pi}{3}, \frac{\pi}{4}$ in rectangular duct without local refinement. 
$u^{h}(\boldsymbol{x})=\sum_{i=0}^{m} p_{i}(\boldsymbol{x}) \alpha_{i}(\boldsymbol{x}) \equiv \mathbf{p}^{T}(\boldsymbol{x}) \mathbf{a}(\boldsymbol{x})$,

where $\mathbf{p}(\boldsymbol{x})$ is a vector of $n$ nodal values (in the present study the monomials up to 2nd order are used, $\left.\mathbf{p}^{T}=\left[\begin{array}{llllll}1 & x & y & x^{2} & x y & y^{2}\end{array}\right]\right), m$ is the number of terms of monomials (polynomial basis), and $a(\boldsymbol{x})$ is a vector of coefficients given by

$$
\mathbf{a}^{T}(\boldsymbol{x})=\left\{\alpha_{0}(\boldsymbol{x}) \alpha_{1}(\boldsymbol{x}) \ldots \alpha_{\mathrm{m}}(\boldsymbol{x})\right\}
$$

which are functions of $\boldsymbol{x}$.

Given a set of $n$ nodal values, of a field function $u_{1}, u_{2}, \ldots, u_{n}$, at $n$ nodes $x_{1}, x_{2}, \ldots, x_{n}$ inside the support domain, Eq. (3.1) can be used for the calculation of the approximated values of the field function at these nodes:

$$
u^{h}\left(\boldsymbol{x}, \boldsymbol{x}_{i}\right)=\mathbf{p}^{T}\left(\boldsymbol{x}_{i}\right) \mathbf{a}(\boldsymbol{x}) \quad i=1,2,3, \ldots, n .
$$

The coefficients a are calculated by the minimization of the quadratic functional $J(\boldsymbol{x})$ given by

$$
J(\boldsymbol{x})=\sum_{i=1}^{n} W\left(\boldsymbol{x}-\boldsymbol{x}_{i}\right)\left[\sum_{j=1}^{m} \mathbf{p}_{j}^{T}\left(\boldsymbol{x}_{i}\right) a(\boldsymbol{x})-u_{i}\right]^{2},
$$

where $W\left(\boldsymbol{x}-\boldsymbol{x}_{i}\right)$ is a weight function.

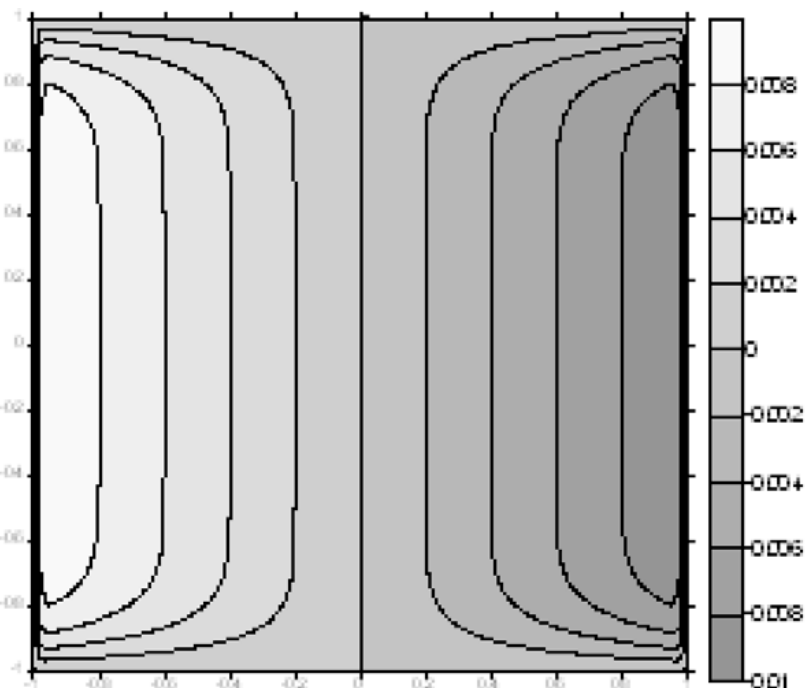

$(\mathrm{B}, \operatorname{tr} \mathrm{ax}=0.0095287, \operatorname{trit1}=-0.0095287)$

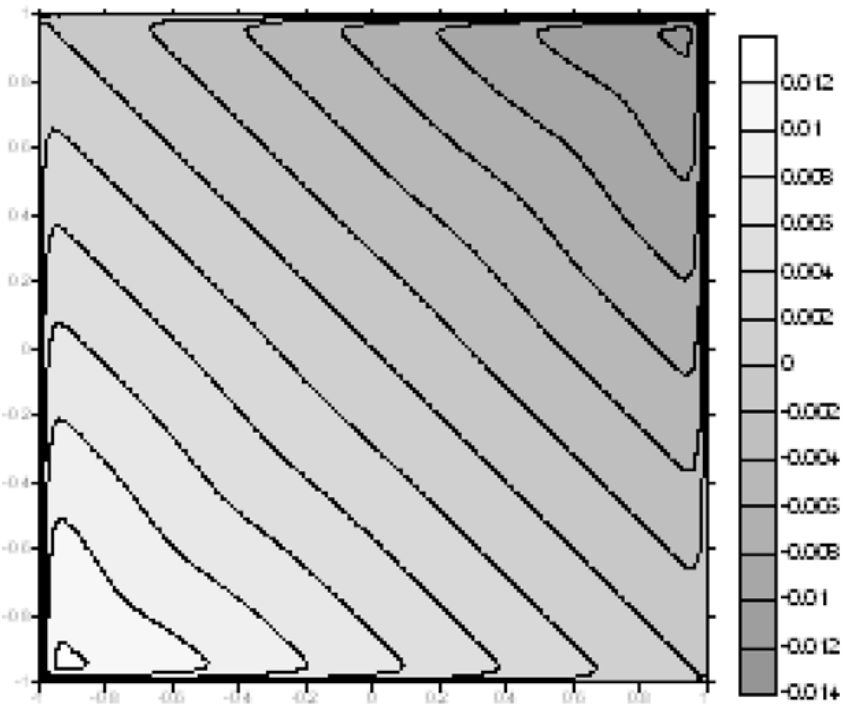

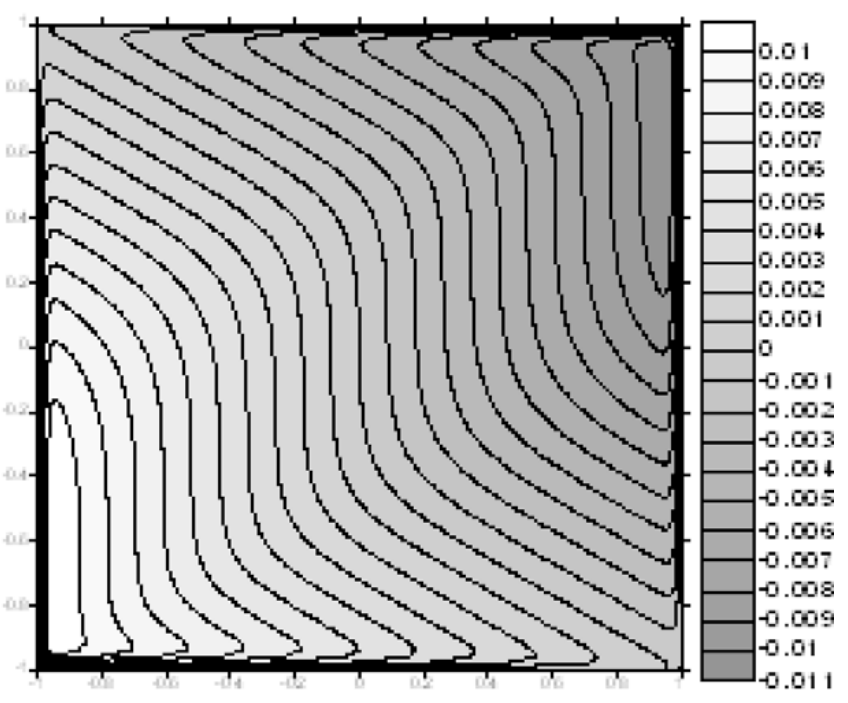

$(\mathrm{B}, \operatorname{tr} \mathrm{ax}=0.010915$, thine $=-0.010915)$

$(\mathrm{B}, \operatorname{tr} \mathrm{ax}=0.0123052, \operatorname{trin}=-0.0123052)$

Fig. 8. Induced magnetic field lines for $M=100$ and $\phi=\frac{\pi}{2}, \frac{\pi}{3}, \frac{\pi}{4}$ in rectangular duct without local refinement. 
The minimization conditions require $\partial J / \partial \mathbf{a}=0$, which results in the following linear equation system:

$$
\mathbf{A}(\boldsymbol{x}) \mathbf{a}(\boldsymbol{x})=\mathbf{B}(\boldsymbol{x}) \mathbf{U}_{s},
$$

where $\mathbf{A}$ is the (weighted) moment matrix, expressed by

$$
\mathbf{A}(\boldsymbol{x})=\sum_{i=1}^{n} \overline{W_{i}}(\boldsymbol{x}) \mathbf{p}(\boldsymbol{x}) \mathbf{p}^{T}\left(\boldsymbol{x}_{i}\right), \quad \bar{W}_{i}(\boldsymbol{x}) \equiv \bar{W}\left(\boldsymbol{x}-\boldsymbol{x}_{i}\right)
$$

In Eq. (3.3), matrix $\mathbf{B}$ has the form $\mathbf{B}(\boldsymbol{x})=\left[\mathbf{B}_{1}, \mathbf{B}_{2}, \ldots, \mathbf{B}_{n}\right]$, where $\mathbf{B}_{i}=\bar{W}_{i}(\boldsymbol{x}) \mathbf{p}\left(\boldsymbol{x}_{i}\right)$ and $\mathbf{U}_{s}$ is the vector that collects the nodal parameters of the field variables for all the nodes in the support domain

$$
\mathbf{U}_{s}=\left\{u_{1}, u_{2}, \ldots, u_{n}\right\}^{T} .
$$

After solving Eq. (3.3) for $\mathbf{a}(\boldsymbol{x})$, one gets

$$
\mathbf{a}(\boldsymbol{x})=\mathbf{A}^{-1}(\boldsymbol{x}) \mathbf{B}(\boldsymbol{x}) \mathbf{U}_{s} .
$$

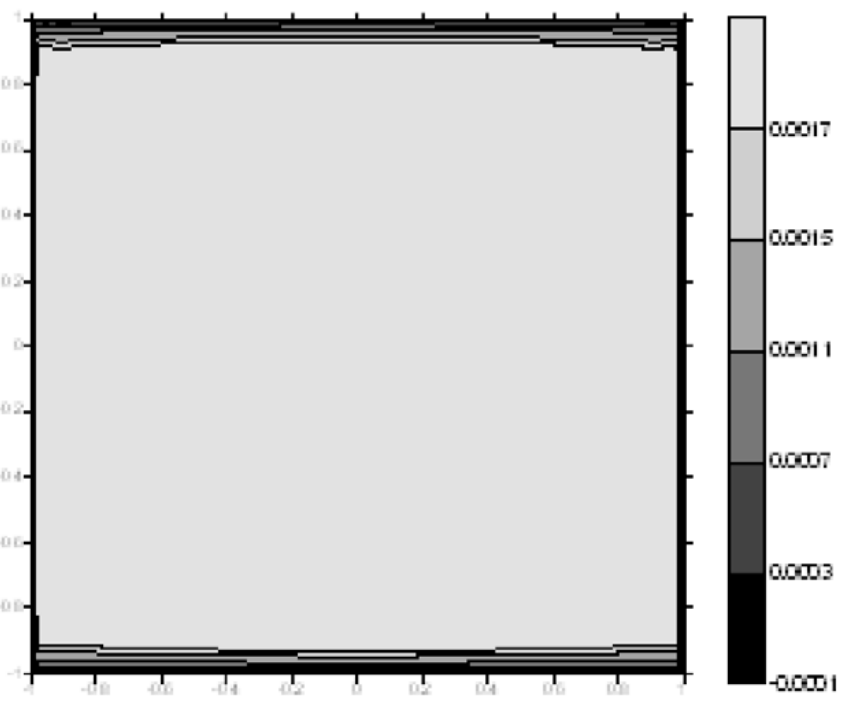

$(u, \max =0.002001, \min =0)$

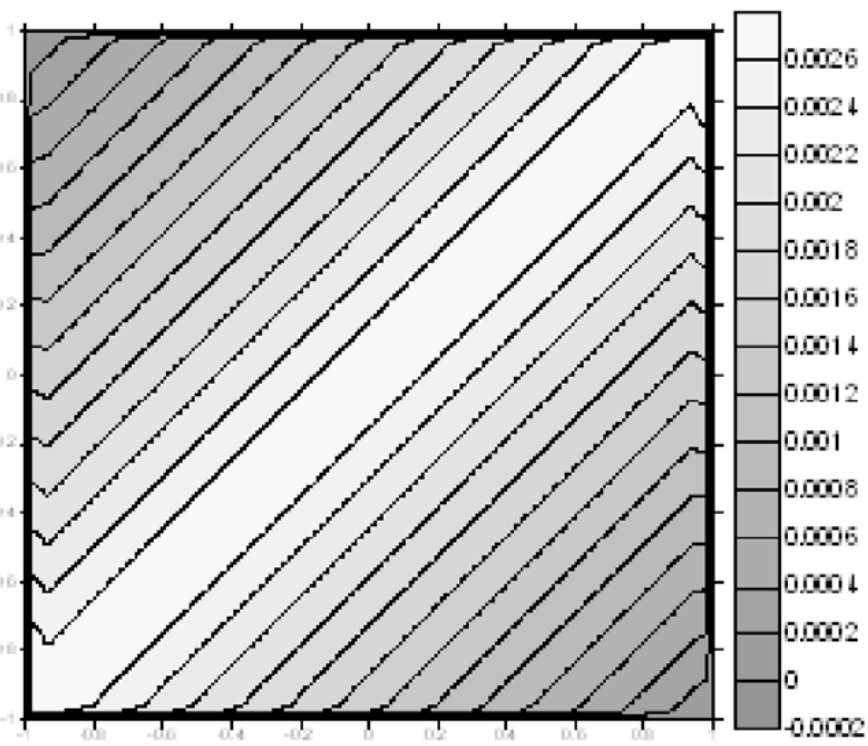

( $u, \operatorname{tr} \mathrm{ax}=0.0028, \operatorname{tritr}=0)$

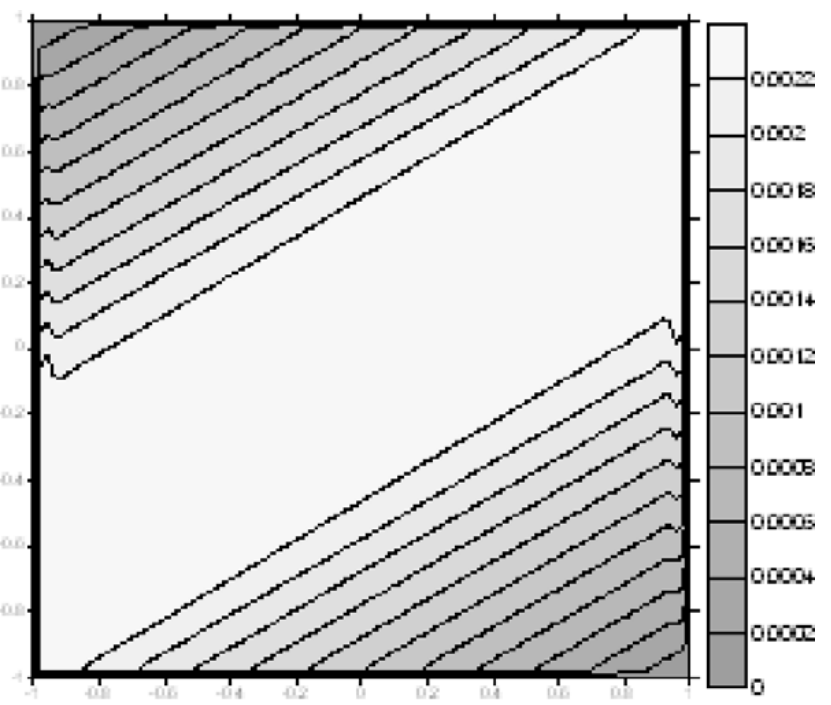

$(u, \max =0.002301, \min =0)$

Fig. 9. Equivelocity lines for $M=500$ and $\phi=\frac{\pi}{2}, \frac{\pi}{3}, \frac{\pi}{4}$ in rectangular duct without local refinement. 
Substitution of last equation in (3.1) leads to

$$
u^{h}(\boldsymbol{x})=\sum_{i=1}^{n} \sum_{j=1}^{m} \mathrm{p}_{j}(\boldsymbol{x})\left(\mathbf{A}^{-1}(\boldsymbol{x}) \mathbf{B}(\boldsymbol{x})\right)_{j i} u_{i} \quad \text { or } \quad u^{h}(\boldsymbol{x})=\sum_{i=1}^{n} \Phi_{i}(\boldsymbol{x}) u_{i}
$$

where the Moving Least Squares shape function $\Phi_{i}(\boldsymbol{x})$ is defined by

$$
\Phi_{i}(\boldsymbol{x})=\sum_{j=1}^{m} \mathrm{p}_{j}(\boldsymbol{x})\left(\mathbf{A}^{-1}(\boldsymbol{x}) \mathbf{B}(\boldsymbol{x})\right)_{j i}=\mathbf{p}^{T} \mathbf{A}^{-1} \mathbf{B}_{i} .
$$

We have to note that $m$ is the number of the monomial terms of the polynomial basis $\mathbf{p}(\boldsymbol{x})$, and $n$ is the number of nodes in the support domain, which are used for constructing the shape function. Moreover, the requirement $n \gg m$ must be fulfilled for the moment matrix $\mathbf{A}$ to be invertible [25].

In order to obtain the spatial derivatives of the approximation function $u^{h}(\boldsymbol{x})$, it is necessary to obtain the derivatives of the MLS shape functions $\Phi_{i}(\boldsymbol{x})$,

$$
\frac{\partial}{\partial \boldsymbol{x}} u^{h}(\boldsymbol{x})=\frac{\partial}{\partial \boldsymbol{x}} \sum_{i=1}^{n} \Phi_{i}(\boldsymbol{x}) u_{i}=\sum_{i=1}^{n}\left\{\frac{\partial}{\partial \boldsymbol{x}} \Phi_{i}(\boldsymbol{x})\right\} u_{i}, \quad \boldsymbol{x}=x, y, z
$$
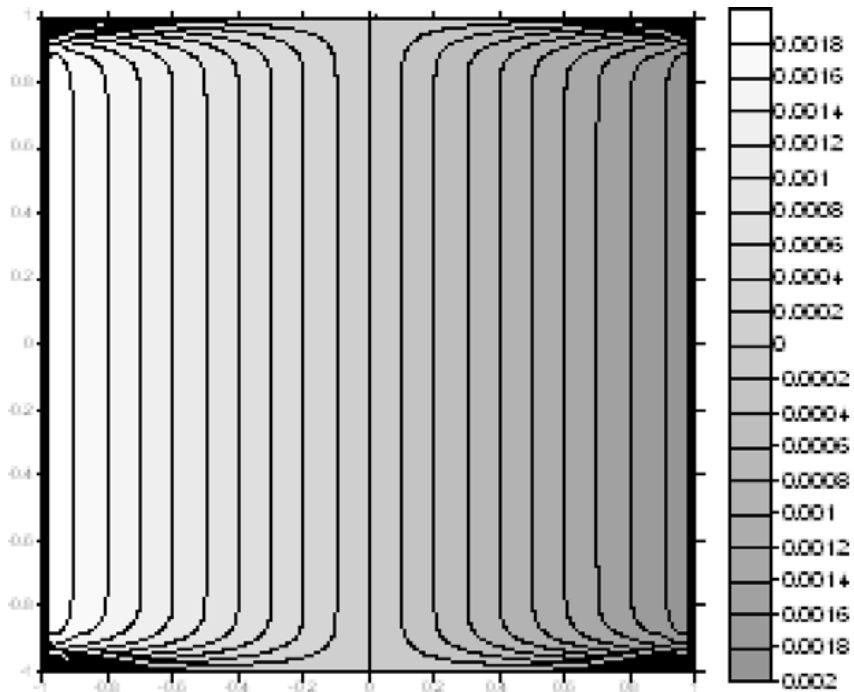

$(B, \operatorname{tr} \alpha x=0.00200, \operatorname{trin}=-0.00200)$

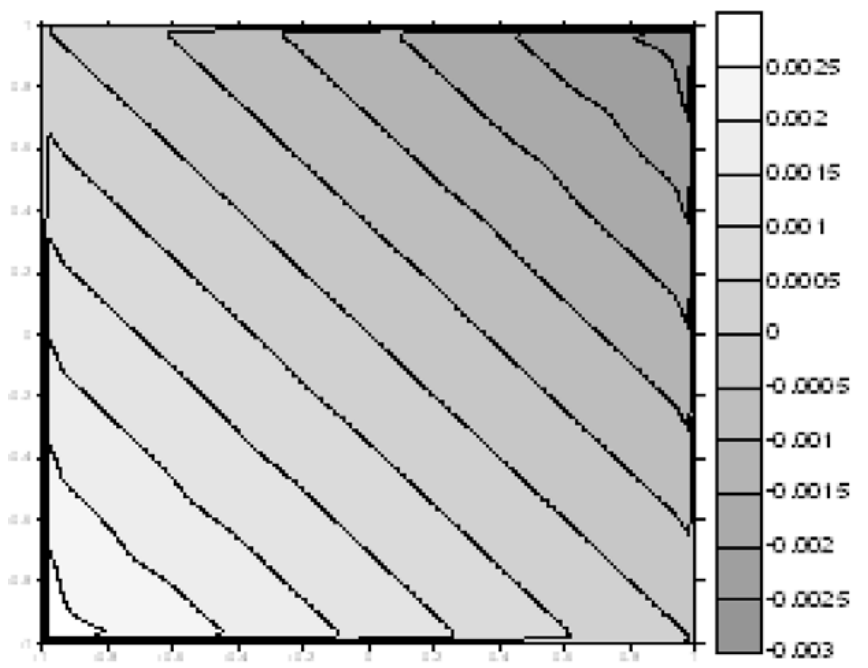

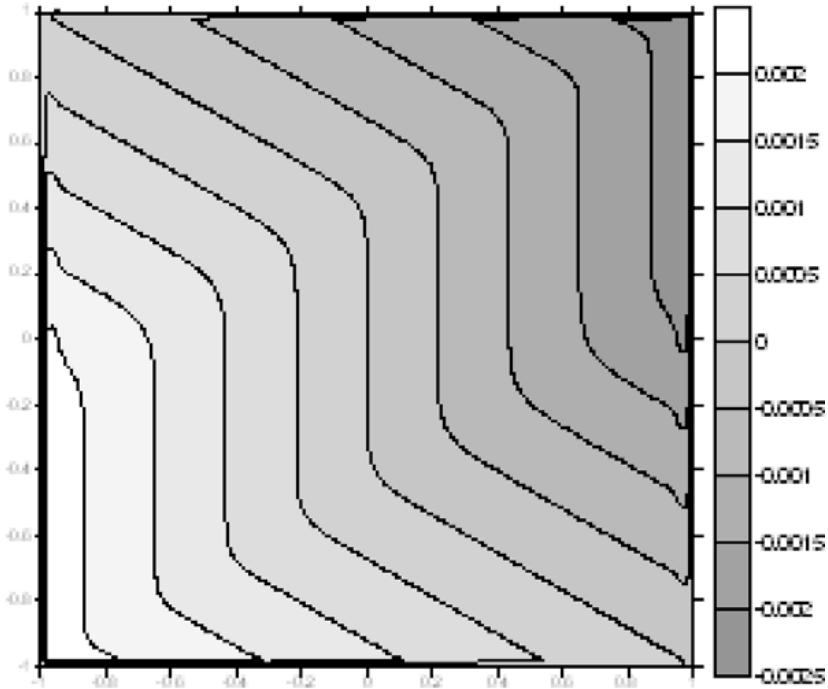

$(B, \max =0.00230, \operatorname{trit}=-0.00230)$

$(B, \operatorname{tn} \operatorname{ax}=0.0030, \operatorname{trin}=-0.0030)$

Fig. 10. Induced magnetic field lines for $M=500$ and $\phi=\frac{\pi}{2}, \frac{\pi}{3}, \frac{\pi}{4}$ in rectangular duct without local refinement. 
The derivative of the shape function is given as

$$
\Phi_{i, \boldsymbol{x}}(\mathbf{x})=\left(\mathbf{p}^{T} \mathbf{A}^{-1} \mathbf{B}_{i}\right)_{, \boldsymbol{x}}=\mathbf{p}_{, x}^{T} \mathbf{A}^{-1} \mathbf{B}_{i}+\mathbf{p}^{T}\left(\mathbf{A}^{-1}\right)_{, \boldsymbol{x}} \mathbf{B}_{i}+\mathbf{p}^{T} \mathbf{A}^{-1}\left(\mathbf{B}_{i}\right)_{, \boldsymbol{x}},
$$

where $\left(\mathbf{A}^{-1}\right)=-\mathbf{A}^{-1}(\boldsymbol{x}) \mathbf{A}(\boldsymbol{x}) \mathbf{A}^{-1}(\boldsymbol{x})$ and comma on the subscript designates a partial derivative with respect to the indicated spatial variable. Regarding the second order derivative of the unknown function one gets

$$
\begin{aligned}
\Phi_{i i, \boldsymbol{x}}(\boldsymbol{x})= & \Phi_{i, \boldsymbol{x}}\left(\Phi_{i, \boldsymbol{x}}(\boldsymbol{x})\right)=\Phi_{i, \boldsymbol{x}}\left(\mathbf{p}_{, \boldsymbol{x}}^{T} \mathbf{A}^{-1} \mathbf{B}_{i}+\mathbf{p}^{T}\left(\mathbf{A}^{-1}\right)_{, \boldsymbol{x}} \mathbf{B}_{i}+\mathbf{p}^{T} \mathbf{A}^{-1}\left(\mathbf{B}_{i}\right)_{, \boldsymbol{x}}\right) \\
= & \left(\mathbf{p}_{, x \boldsymbol{x}}^{T} \mathbf{A}^{-1} \mathbf{B}_{i}+\mathbf{p}_{, \boldsymbol{x}}^{T}\left(\mathbf{A}^{-1}\right)_{, \boldsymbol{x}} \mathbf{B}_{i}+\mathbf{p}_{, x}^{T} \mathbf{A}^{-1}\left(\mathbf{B}_{i}\right)_{, \boldsymbol{x}}\right)+\left(\mathbf{p}_{, \boldsymbol{x}}^{T}\left(\mathbf{A}^{-1}\right)_{, \boldsymbol{x}} \mathbf{B}_{i}+\mathbf{p}_{, \boldsymbol{x}}^{T}\left(\mathbf{A}^{-1}\right)_{, \boldsymbol{x} x} \mathbf{B}_{i}+\mathbf{p}_{, \boldsymbol{x}}^{T}\left(\mathbf{A}^{-1}\right)_{, \boldsymbol{x}}\left(\mathbf{B}_{i}\right)_{, \boldsymbol{x}}\right) \\
& +\left(\mathbf{p}_{, x}^{T} \mathbf{A}^{-1}\left(\mathbf{B}_{i}\right)_{, \boldsymbol{x}}+\mathbf{p}^{T} A_{\boldsymbol{x}}^{-1}\left(\mathbf{B}_{i}\right)_{, \boldsymbol{x}}+\mathbf{p}^{T} A_{\boldsymbol{x}}^{-1}\left(\mathbf{B}_{i}\right)_{, \boldsymbol{x} x}\right),
\end{aligned}
$$

where $\boldsymbol{x}=x, y, z$ and $\left(\mathbf{A}^{-1}\right)_{, \boldsymbol{x} x}=-\left(\mathbf{A}^{-1}\right)_{, \boldsymbol{x}} \mathbf{A} \mathbf{A}^{-1}-\mathbf{A}^{-1} \mathbf{A}_{, \boldsymbol{x}} \mathbf{A}^{-1}-\mathbf{A}^{-1} \mathbf{A}\left(\mathbf{A}^{-1}\right)_{, \boldsymbol{x}}$.

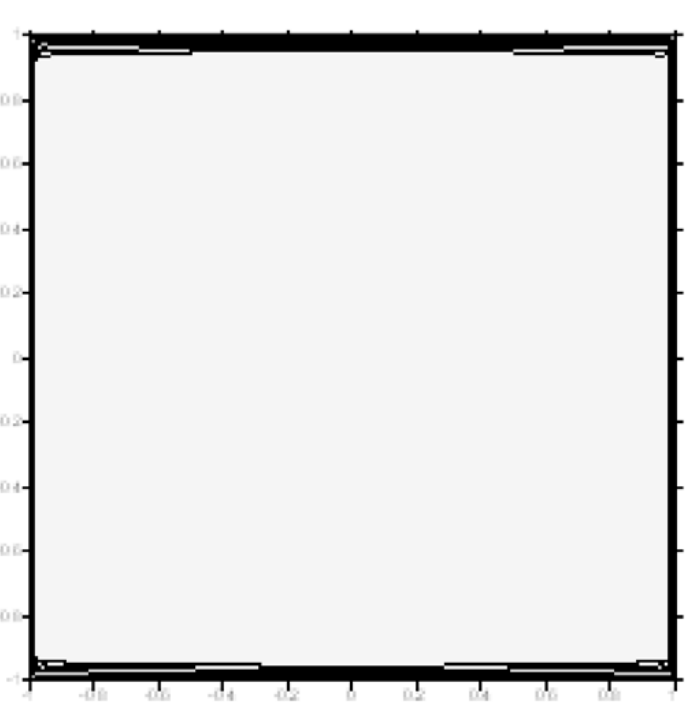

$(u, \max =0.001, \min =0)$

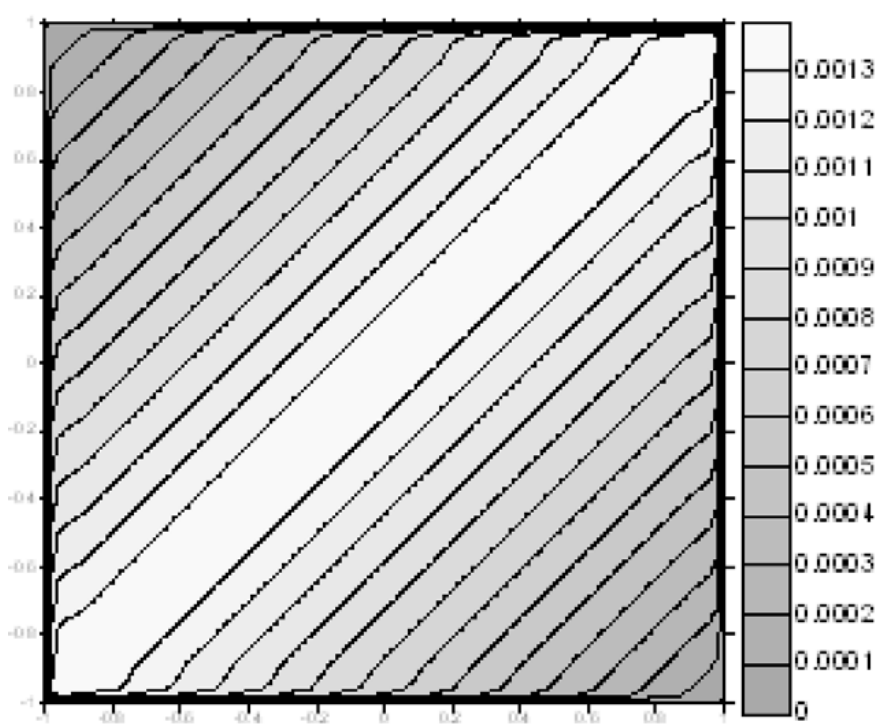

$(u, \max =0.001346, \operatorname{mit}=0)$

Fig. 11. Equivelocity lines for $M=1000$ and $\phi=\frac{\pi}{2}, \frac{\pi}{3}, \frac{\pi}{4}$ in rectangular duct without local refinement. 


\subsection{Weight function}

The weight function is non-zero over a small neighborhood of $\boldsymbol{x}_{i}$, called the support domain of node $i$. The choice of the weight function $W\left(\boldsymbol{x}-\boldsymbol{x}_{i}\right)$ affects the resulting approximation $u^{h}\left(\boldsymbol{x}_{i}\right)$ significantly. In the present paper a Gaussian weight function is used [25,26], Fig. 2, yet the support domain does not have a standard point density value. Instead, a constant number of nodes are used for the approximation of the field function (Fig. 3 ).

$$
\bar{W}\left(\boldsymbol{x}-\boldsymbol{x}_{i}\right) \equiv \bar{W}(\bar{d})=\left\{e^{-\left(\frac{\bar{d}_{l}}{a}\right)^{2}} \begin{array}{c}
0 \\
0
\end{array},\right.
$$

where $I=1,2,3, \ldots, q$ are the nodes that produce the support domain of node $\boldsymbol{x}_{i}$, and $\bar{d}=\frac{\left|\boldsymbol{x}-\boldsymbol{x}_{i}\right|}{a_{0}^{2}}$ with $a_{0}$ a prescribed constant (often $a_{0}=0.3$ ).

\subsection{System equation discretization}

The Meshless Point Collocation method is a MFree "strong-form" description method. In these methods the "strong-form" description of the governing equations and the boundary conditions are used and they are discretized by collocation tech-

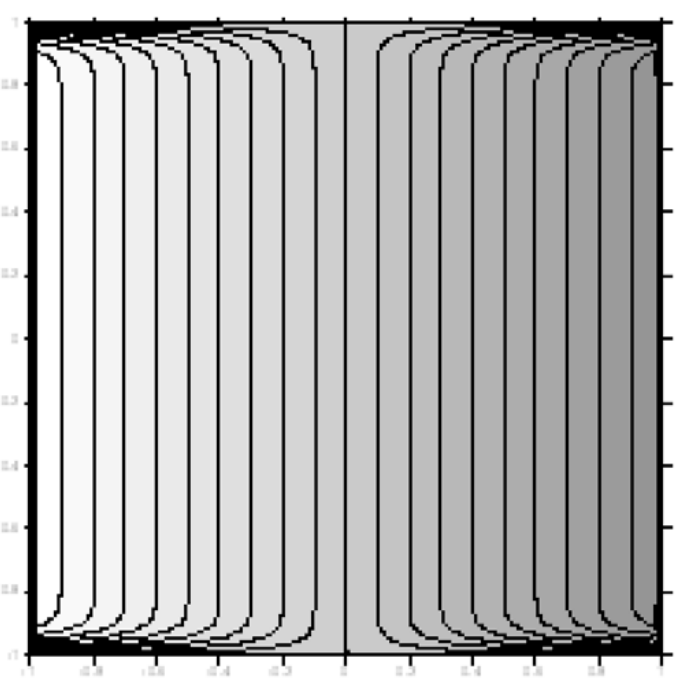

$(B, \operatorname{trax}=0.001, \operatorname{tritr}=-0.001)$

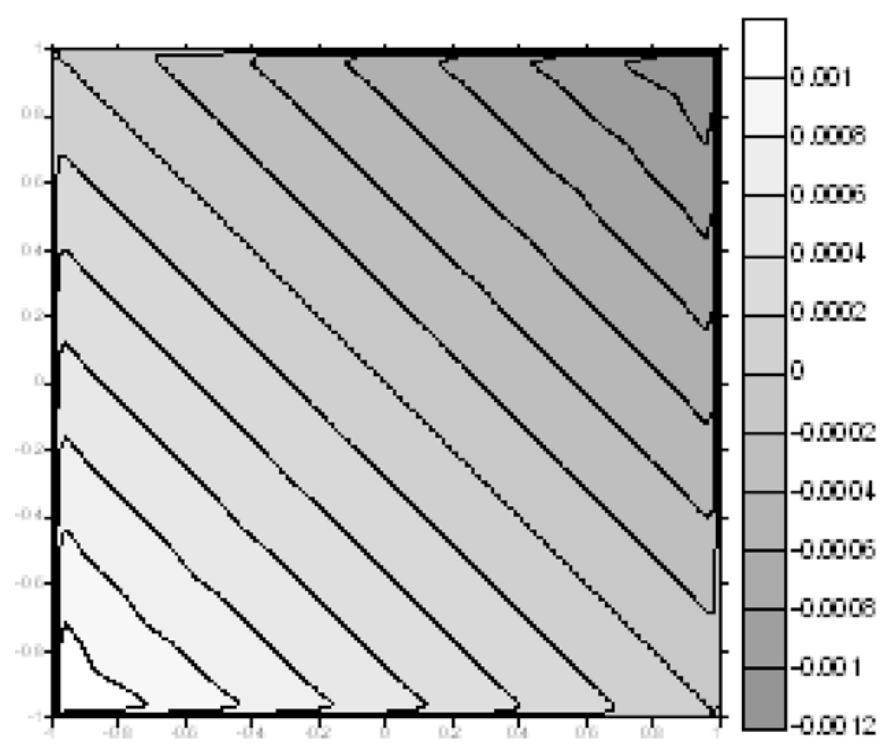

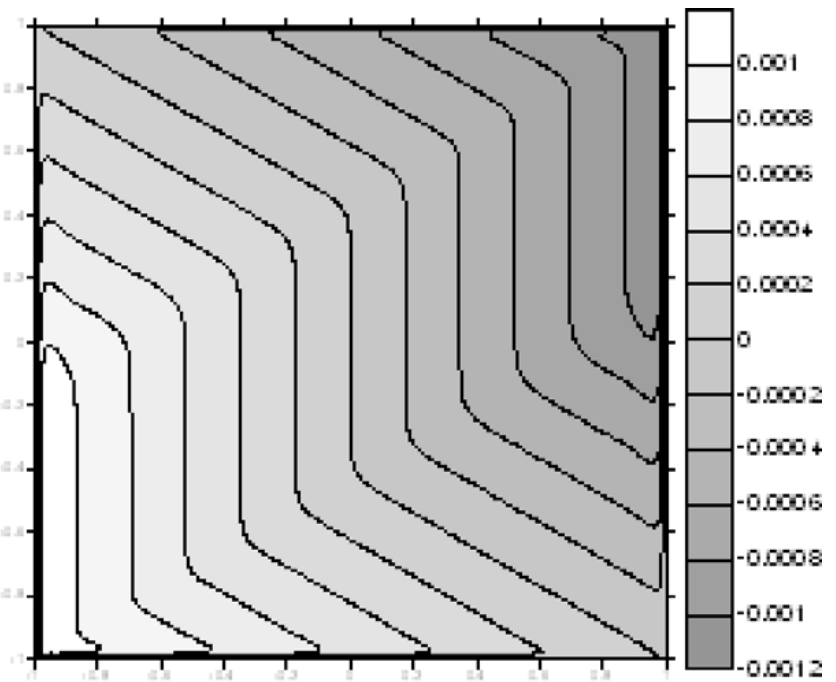

$(B, \operatorname{thax}=0.001, \operatorname{trit}=-0.001)$

$(B, \operatorname{tr} \alpha x=0.001066, \operatorname{tritr}=-0.001066)$

Fig. 12. Induced magnetic field lines for $M=1000$ and $\phi=\frac{\pi}{2}, \frac{\pi}{3}, \frac{\pi}{4}$ in rectangular without local refinement. 
niques. The MFree strong-form methods possess the following attractive advantages. They are truly meshless and the implementing procedure is straightforward, while the algorithms and the implementation can be kept simple, particularly when handling problems with Dirichlet boundary conditions only. Under these conditions, these methods are highly computationally efficient, even with polynomial approximation functions, and the solution can be systematically obtained with increased accuracy, compared to FEM, FDM, or other CFD methods. In general, MFree strong-form methods may still suffer from some local stability and accuracy issues, depending on the problem [25]. However, these local restrictions are now systematically avoided with the utilization of Type-I nodal distribution and proper local point cloud refinement procedures, in accordance with $[24,26]$, even for natural or mixed type boundary conditions.

Collocation method using MLS may be considered as a special case of the "weak-form" methods [27]. Moreover, this collocation method may be considered as a "weak-solution", with a Dirac delta function as the test (weight) function [28,29]. The weighted residual method provides a flexible mathematical framework for the construction of a variety of numerical solution schemes for the differential equations arising in the field of both science and engineering. Its application, in conjunction with the Moving Least Square (MLS) approximation method, yields powerful solution algorithms for the governing equations.
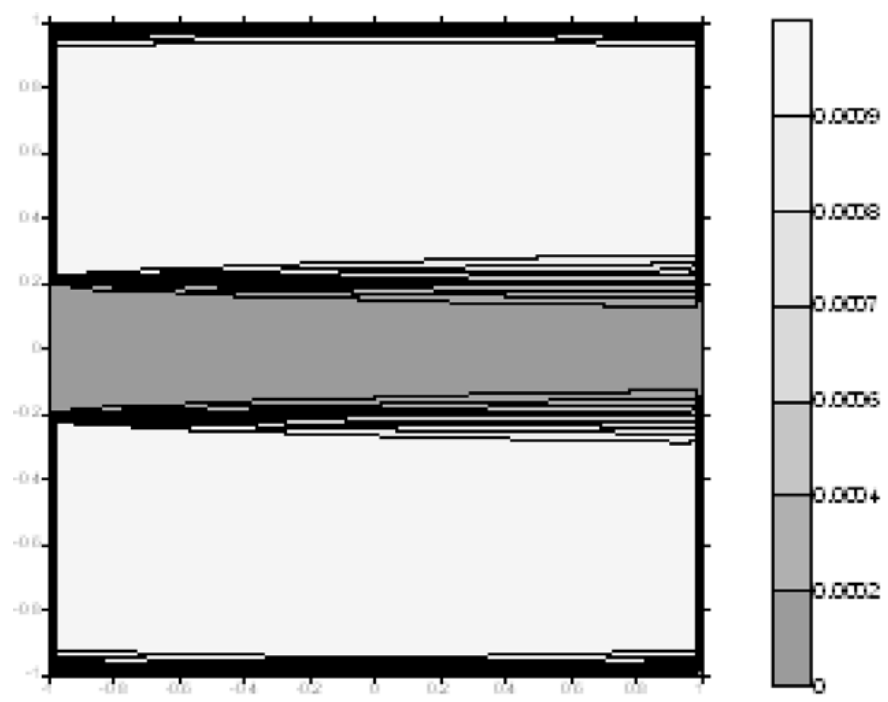

$(u, \max =0.001, \min =0)$

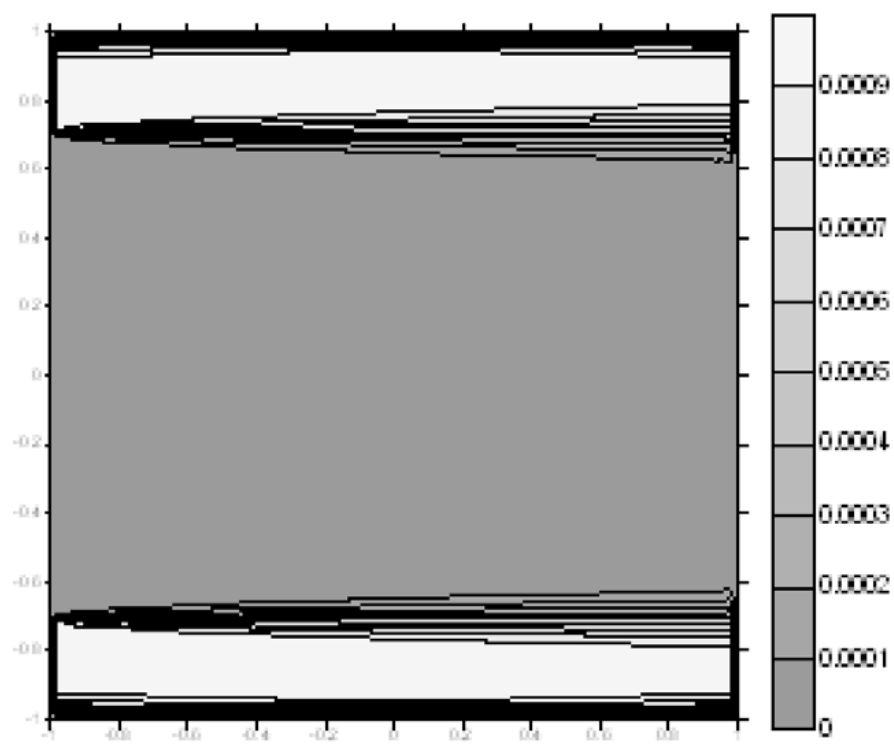

$(u, \operatorname{tn} \alpha=0.001$, thitr $=0)$

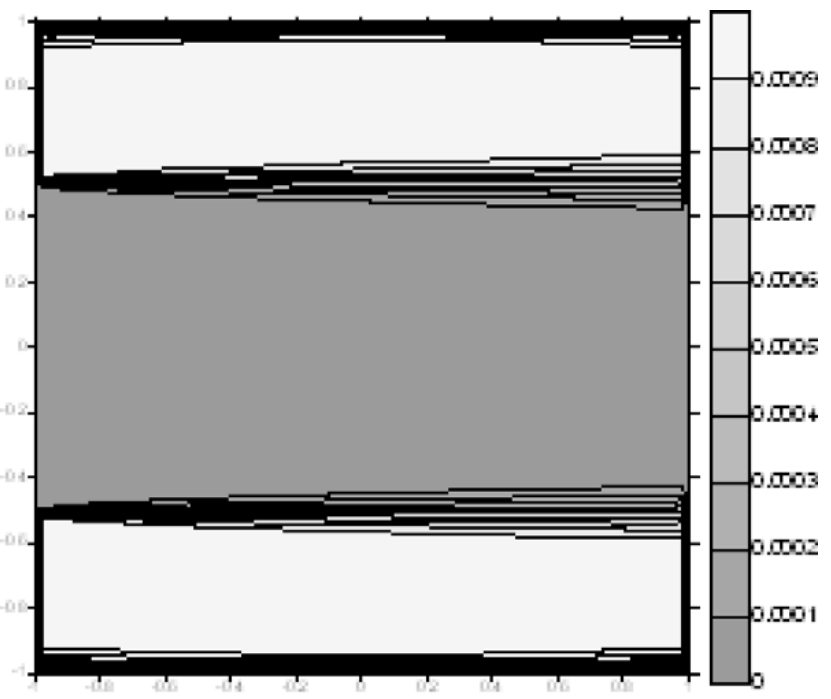

$(u \max =0.001, \min =0)$

Fig. 13. Equivelocity lines for $M=1000, \phi=\frac{\pi}{2}, L=0.2,0.5,0.7$ in rectangular duct without local refinement. 
Considering our problem (Case 1) governed by the differential equations

$$
\begin{aligned}
& \nabla^{2} u+M \frac{\partial B}{\partial x}=-1=f_{1}, \\
& \nabla^{2} B+M \frac{\partial u}{\partial x}=0=f_{2}, \quad \text { in } \quad \Omega .
\end{aligned}
$$

The boundary conditions in the first case can be expressed as $u=u_{p}$ on $\partial \Omega, B=B_{p}$ on $\Gamma_{u}$ and $\frac{\partial B}{\partial \mathbf{n}}=t$ on $\Gamma_{t}$, studied over the domain $\Omega$, which is a sufficiently smoothed, closed, and surrounded by a continuous boundary $\partial \Omega=\Gamma_{u} \cup \Gamma_{t}$. In Eqs. (3.4) and (3.5), $u(x, y)$ and $B(x, y)$ are the dependent variables of the problem (functions of independent spatial variables), $u_{p}$ and $B_{p}$ are the prescribed value of the unknown functions over the boundary $\partial \Omega$ and $\Gamma_{u}$, while $f_{1}, f_{2}$ and $t$ are the forces and the source or sink terms acting over the domain $\Omega$ and the boundary $\Gamma_{t}$, respectively. In the absence of an exact analytical solution for Eqs. (3.4) and (3.5), one may seek to represent the field variables $u(x, y)$ and $B(x, y)$ approximately as

$$
u^{h}(\boldsymbol{x})=\sum_{i=1}^{n} \Phi_{i}(\boldsymbol{x}) u_{i} \quad B^{h}(\boldsymbol{x})=\sum_{i=1}^{n} \Phi_{i}(\boldsymbol{x}) B_{i}
$$

$u_{i}$ and $B_{i}$ are two sets of coefficients (constants), which are the nodal unknowns, whereas $\Phi_{i}$ represent a set of geometrical functions, usually called shape functions. Accuracy and convergence of the defined approximation will depend on the
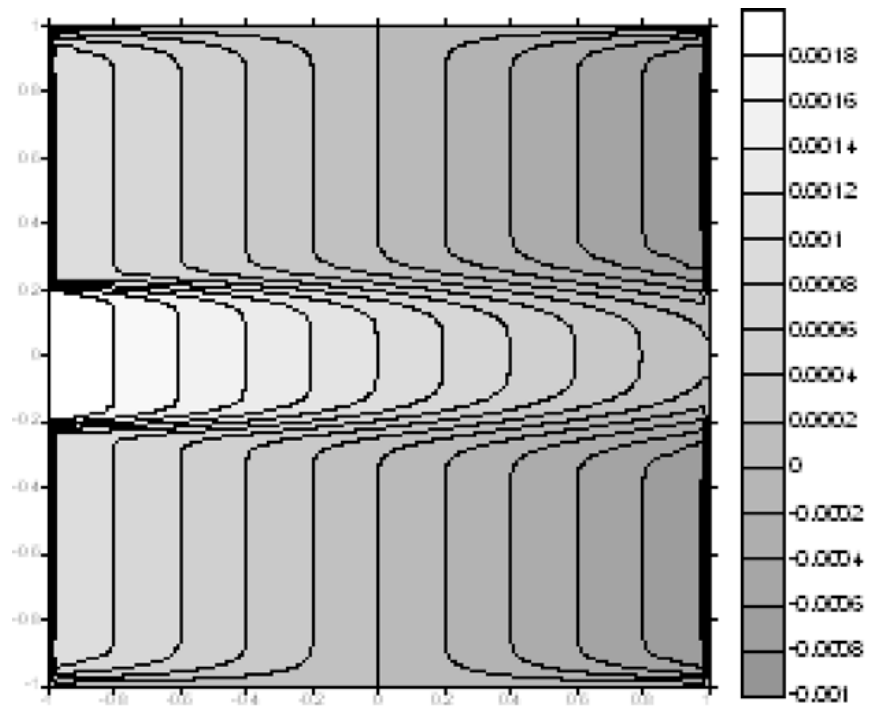

(B, $\max =0.00199$, mirr $=-0.0010$ )

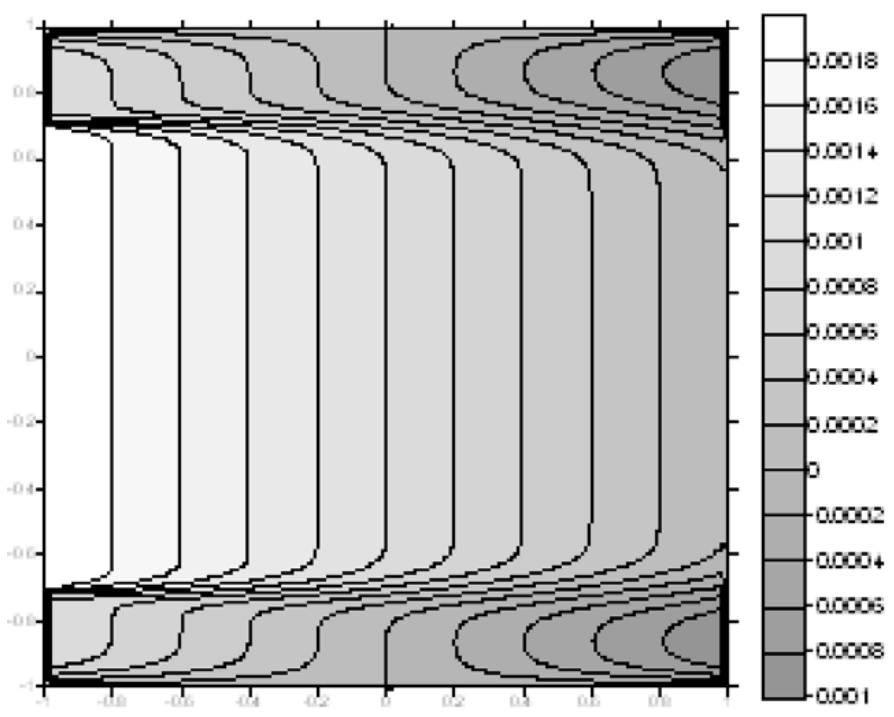

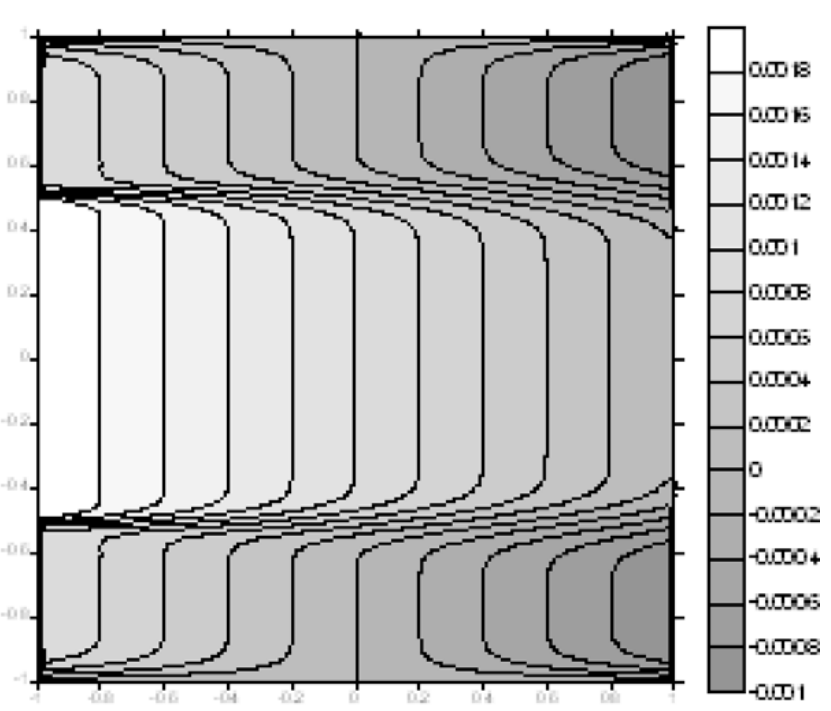

(B, $\max =0.00199, \operatorname{mir}=-0.0010)$

$(B, \max =0.00199, \min =-0.0010)$

Fig. 14. Induced magnetic field lines for $M=1000, \phi=\frac{\pi}{2}, L=0.2,0.5,0.7$ in rectangular duct without local refinement. 
selected basis functions and (as a rule of thumb) these functions should be chosen in a way that the approximation gradually becomes more accurate as $m$ increases. Substitution of Eq. (3.6) into Eqs. (3.4) and (3.5) gives

$$
\begin{aligned}
& \nabla^{2} u^{h}+M \frac{\partial B^{h}}{\partial x}-f_{1}=R_{1, \Omega}, \\
& \nabla^{2} B^{h}+M \frac{\partial u^{h}}{\partial x}-f_{2}=R_{2, \Omega},
\end{aligned}
$$

where $R_{1, \Omega}$ and $R_{2, \Omega}$ are the residuals that appear through the insertion of an approximation instead of an exact solution for the unknown functions $u(x, y)$ and $B(x, y)$.

The residuals $R_{1, \Omega}$ and $R_{2, \Omega}$ are a function of position inside $\Omega$. The weighted residual method is based on the minimization of the residuals over the entire domain. For this minimization procedure to be achieved the residuals are weighted by an appropriate number of position-dependent functions and a summation is carried out. The latter is written

$$
\begin{aligned}
& \int_{\Omega} W_{i} R_{1, \Omega} \mathrm{d} \Omega=0, \quad i=1,2,3, \ldots, n, \\
& \int_{\Omega} W_{i} R_{2, \Omega} \mathrm{d} \Omega=0, \quad i=1,2,3, \ldots, n,
\end{aligned}
$$

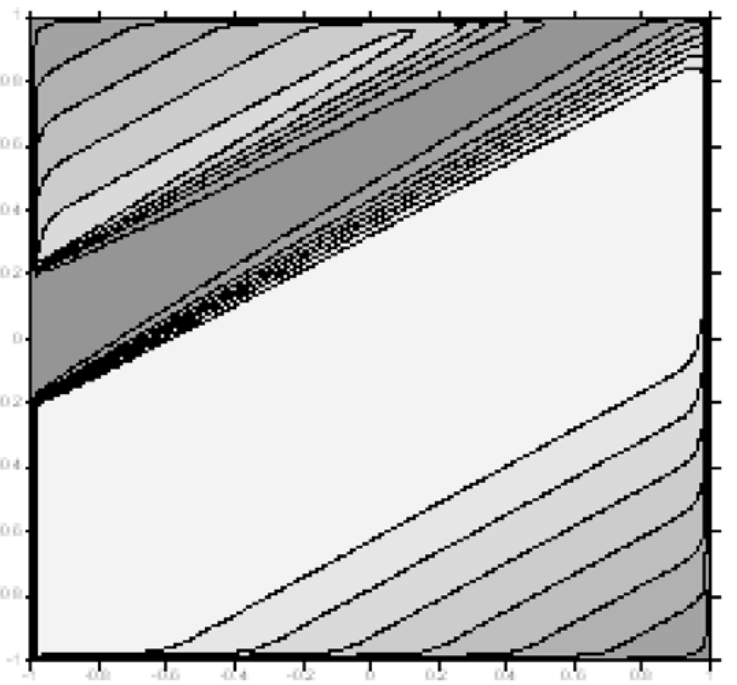

$(u, \max =0.00199, \min =-0.0010)$

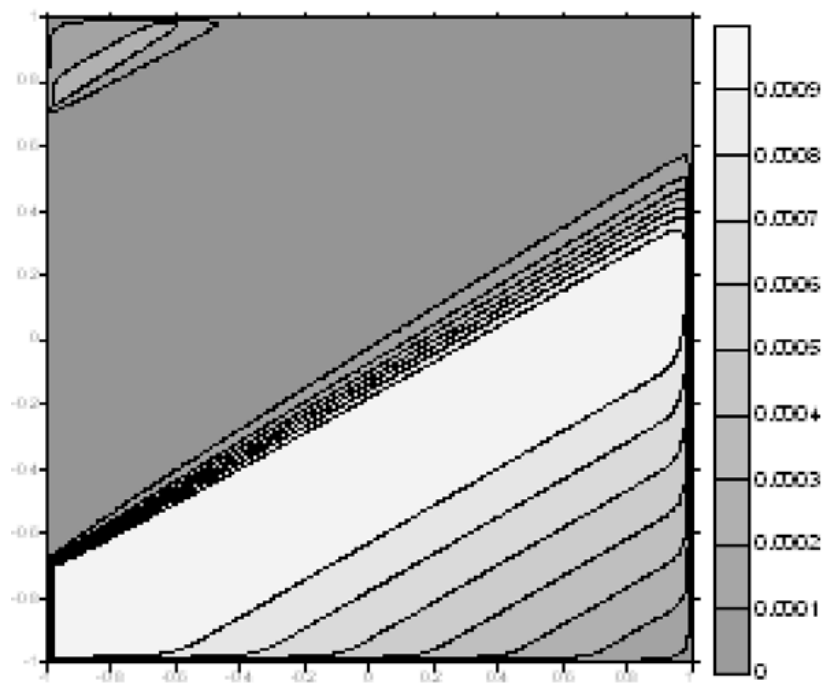

$(u, \max =0.00199, \operatorname{tritr}=-0.0010)$
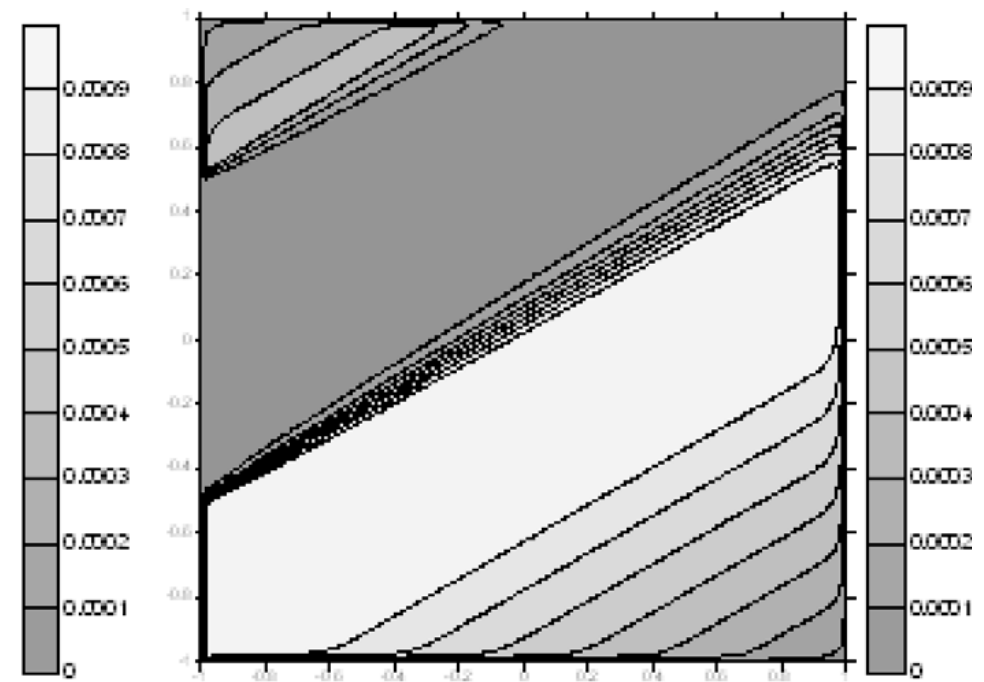

$(u, \max =0.00199, \min =-0.0010)$

Fig. 15. Equivelocity lines for $M=1000, \phi=\frac{\pi}{3}, L=0.2,0.5,0.7$ in rectangular duct local refinement. 
where $W_{i}$ are the independent weight functions and $\mathrm{d} \Omega$ is an appropriate integration interval. Applying the weighted residual method to the above equations one gets

$$
\begin{aligned}
& \int_{\Omega} W_{i}\left(\nabla^{2} u^{h}+M \frac{\partial B^{h}}{\partial x}-f_{1}\right) \mathrm{d} \Omega+\int_{\Omega} W_{i}\left(\nabla^{2} B^{h}+M \frac{\partial u^{h}}{\partial x}-f_{2}\right) \mathrm{d} \Omega+\int_{\partial \Omega} W_{i}^{\partial \Omega}\left(u^{h}-u_{p}\right) \mathrm{d} \partial \Omega \\
& +\int_{\Gamma_{u}} W_{i}{ }^{\Gamma_{u}}\left(B^{h}-B_{p}\right) \mathrm{d} \Gamma_{u}+\int_{\Gamma_{t}} W_{i} \Gamma_{t}\left(\frac{\partial B^{h}}{\partial \mathbf{n}}-t\right) \mathrm{d} \Gamma_{t}=0,
\end{aligned}
$$

with the weighted functions $W_{i}, W_{i}^{\partial \Omega}, W_{i}^{\Gamma_{u}}, W_{i}^{\Gamma_{\mathrm{t}}}$ defined in appropriate ways. Theoretically, the above equation should provide a system

$$
\boldsymbol{K u}=\boldsymbol{f}
$$

of $n$ linear equations to be solved, in order to calculate the coefficients $u_{i}$ and $B_{i}$ in Eq. (3.6).

In cases where $W_{i} \equiv \delta_{i}, \delta_{i}$ being the Dirac delta function, Eqs. (3.9) and (3.10) can be written:

$$
\begin{aligned}
& \nabla^{2} u_{i}^{h}+M \frac{\partial B_{i}^{h}}{\partial x}=-1=f_{1}, \\
& \nabla^{2} B_{i}^{h}+M \frac{\partial u_{i}^{h}}{\partial x}=0=f_{2}, \quad i \in \Omega .
\end{aligned}
$$
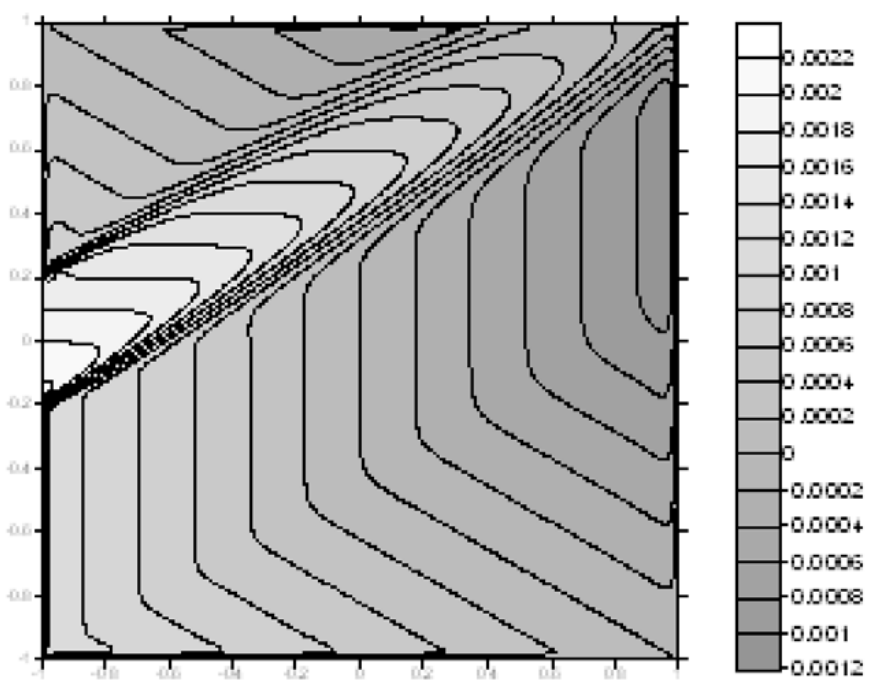

$(B, \max =0.00199, \operatorname{trin}=-0.0010)$

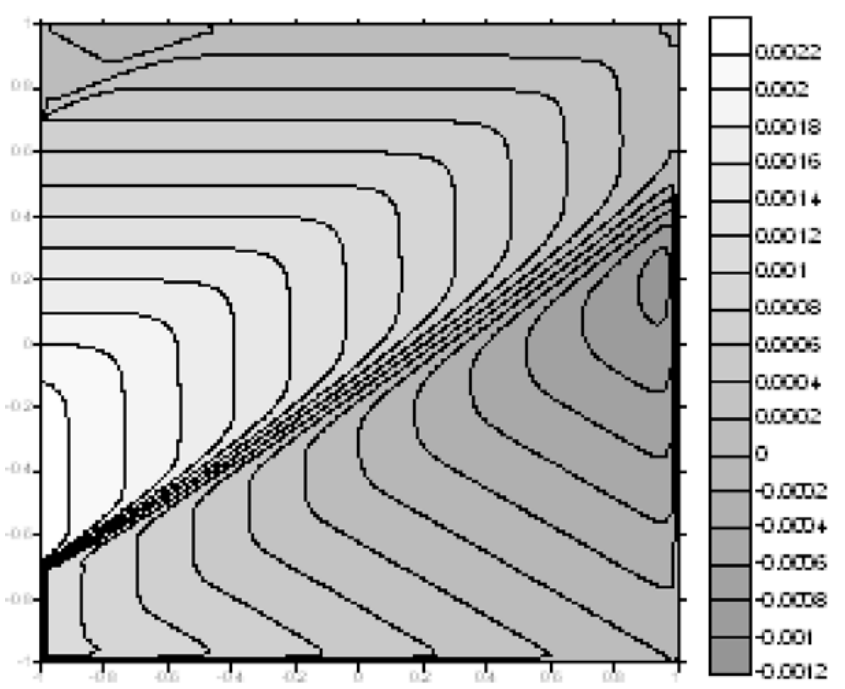

$(B, \operatorname{tr} \mathrm{ax}=0.00199$, thitr= -0.0010$)$

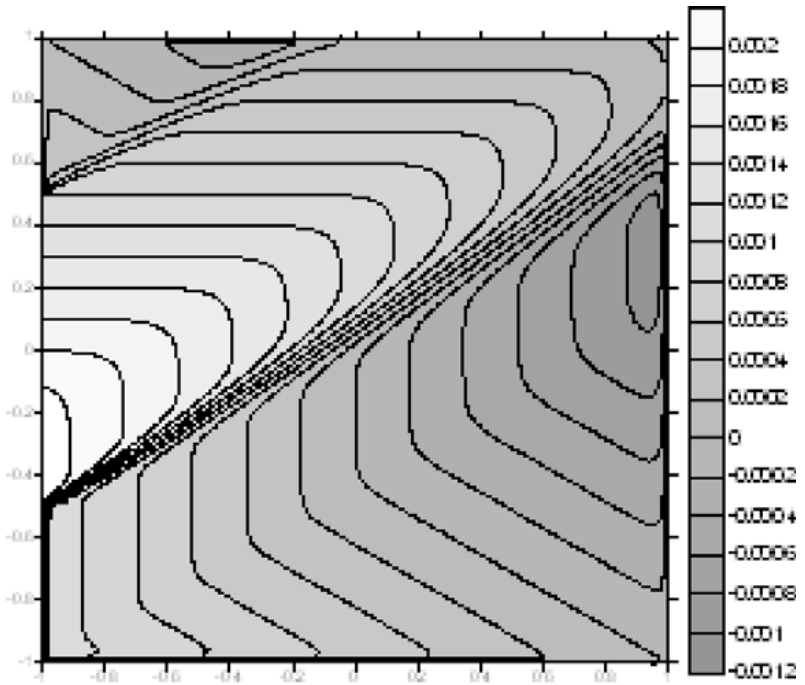

$(B, \max =0.00199, \min =-0.0010)$

Fig. 16. Induced magnetic field lines for $M=1000, \phi=\frac{\pi}{3}, L=0.2,0.5,0.7$ in rectangular duct without local refinement. 
The boundary conditions in the first case can be expressed as

$$
\begin{aligned}
& u_{j}^{h}=u_{p} \quad j \in \partial \Omega, \\
& B_{k}^{h}=B_{p}, \quad k \in \Gamma_{u} \\
& \frac{\partial B_{g}^{h}}{\partial \mathbf{n}}=t, \quad g \in \Gamma_{t} .
\end{aligned}
$$

leading to a linear system of the form $\boldsymbol{L u}=\boldsymbol{f}$, where the matrix operator $\boldsymbol{L}$ contains the derivative operators appearing in the MHD flow equations.

\section{Results and discussion}

\subsection{Rectangular duct}

Numerical experiments have been performed for a viscous, incompressible and electrically conducting fluid, flowing in the $z$-direction along a duct which has either a rectangular, a circular, an elliptical, or an arbitrary cross-section in the $x y$ plane. Through its passage it is subjected to a constant and uniform magnetic field $\mathbf{B}_{0}$ aligned onto the $x y$-plane. Thus,

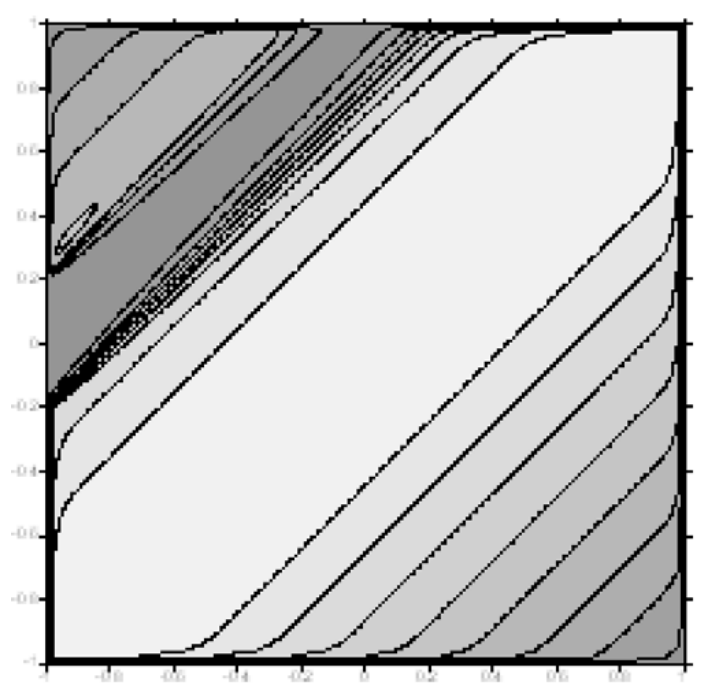

$(u, \max =0.00199, \min =-0.0010)$

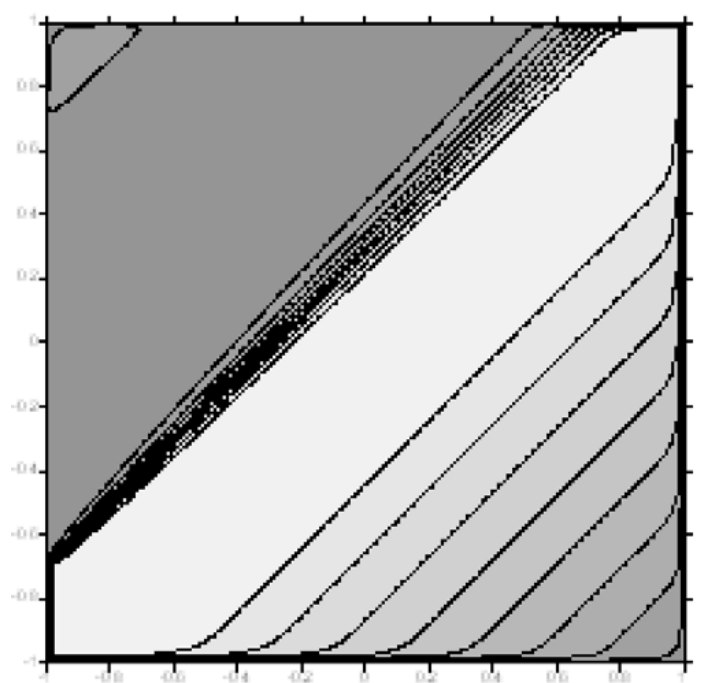

$(u, \operatorname{th} \mathrm{ax}=0.00199, \operatorname{mitr}=-0.0010)$
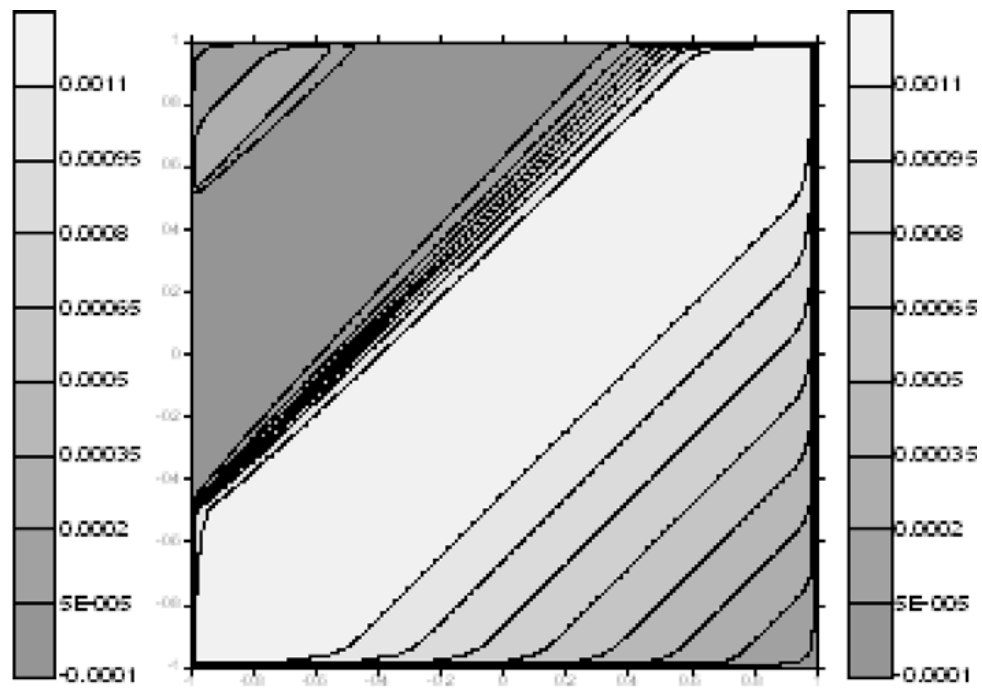

$(u, \max =0.00199, \min =-0.0010$

Fig. 17. Equivelocity lines for $M=1000, \phi=\frac{\pi}{4}, L=0.2,0.5,0.7$ in rectangular duct without local refinement. 
the problem is a two-dimensional MHD flow problem and the $z$-components of the velocity and induced magnetic field are $u(x, y)$ and $B(x, y)$, respectively.

First, the MHD duct problem is solved in a duct with a square cross-section. The domain and the boundary of the square region $(|x| \leqslant 1,|y| \leqslant 1)$ are discretized using node distribution of Type I [22,24]. The Hartmann number $M$ ranges from small $(M=5)$ to moderate $(M=500)$ and to large values $\left(M=10^{4}\right.$ up to $\left.10^{5}\right)$, while the number of the nodes ranges approximately from $10^{3}$ to $10^{4}$, without using refinement procedures. Eqs. (2.5) and (2.6) or (2.7) and (2.8) clearly resemble the convection-diffusion equations. Thus, when the Hartmann number $M$ increases, the convection term is dominant, and, thus, boundary layers are emerging. Local refinement by the proper addition of interstitial nodes near those points where $|\boldsymbol{L u}-\boldsymbol{f}|<r, r$ being a predefined small number (e.g. $r=10^{-5}$ ), makes the solution stable, accurate, and fast converging.

Herein, we present four characteristic test cases, each with varying boundary conditions.

Case 1: Duct with insulating walls

For the case of a rectangular duct with a square cross section $(|x| \leqslant 1,|y| \leqslant 1)$, subjected to a magnetic field in the $x$-direction (i.e. $\phi=\pi / 2$ ), the MHD flow equations are given from Eqs. (2.5) and (2.6). The walls of the duct are insulating $(B=0)$ and the velocity is zero on the solid walls $(u=0)$. Coupled Eqs. (2.5) and (2.6) are expressed in matrix differential operator form $\boldsymbol{L} \boldsymbol{u}=\boldsymbol{f}$, where

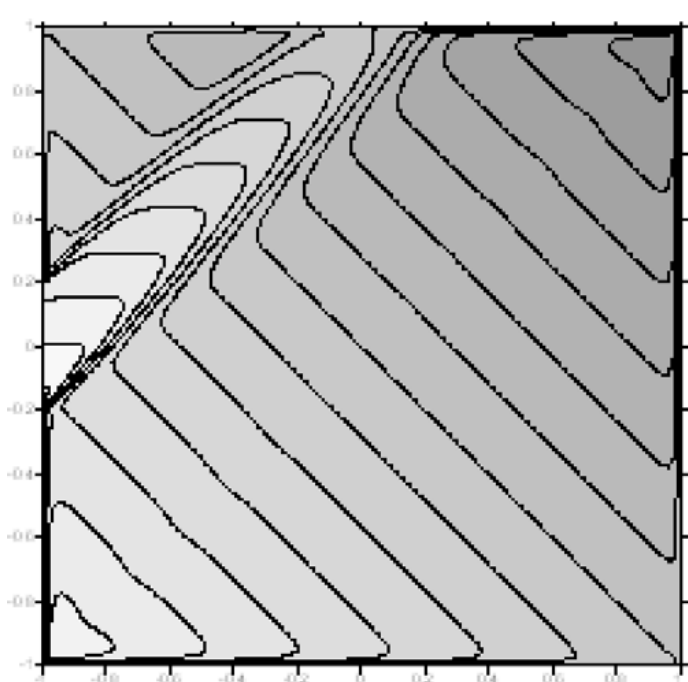

$(B, \max =0.00157, \operatorname{trit}=-0.00134)$

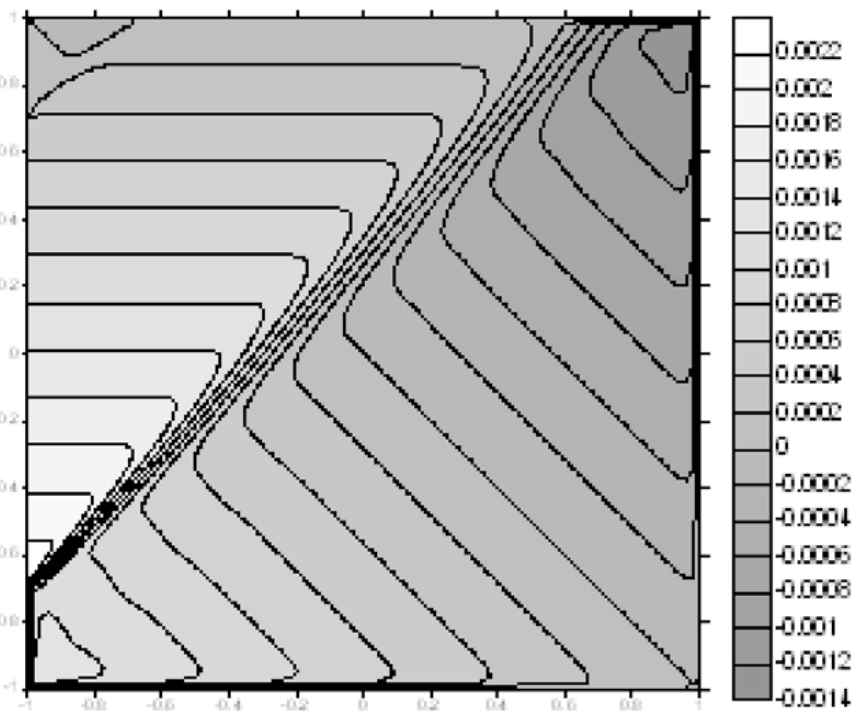

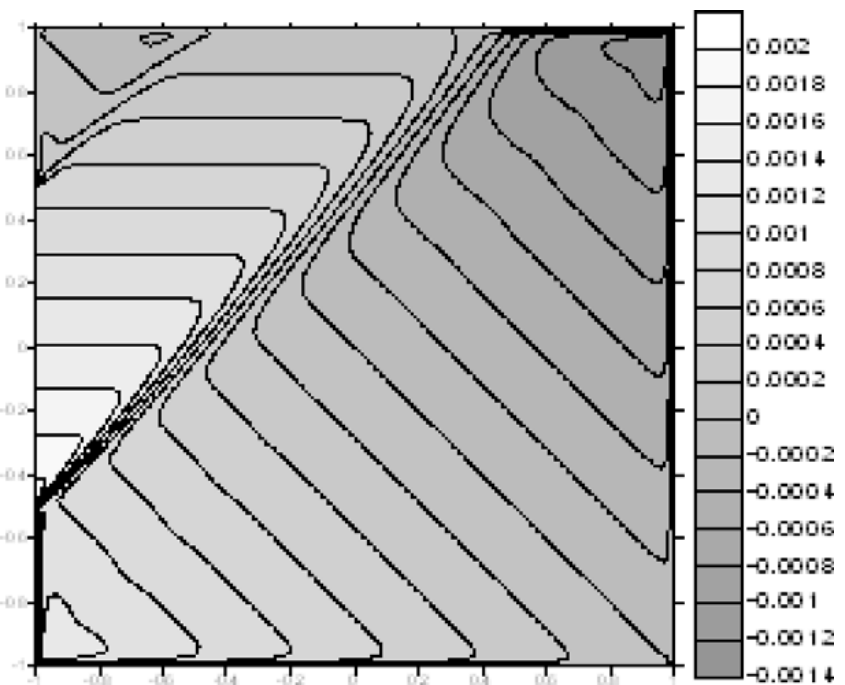

$(B, \operatorname{trax}=0.002, \operatorname{trin}=-0.0014)$

$(B, \max =0.0024, \operatorname{trit}=-0.0014)$

Fig. 18. Induced magnetic field lines for $M=1000, \phi=\frac{\pi}{4}, L=0.2,0.5,0.7$ in rectangular duct without local refinement. 


$$
\mathbf{L}=\left[\begin{array}{cc}
\nabla^{2} & M \frac{\partial}{\partial x} \\
M \frac{\partial}{\partial x} & \nabla^{2}
\end{array}\right], \quad \mathbf{u}=\left[\begin{array}{l}
u \\
B
\end{array}\right], \quad \mathbf{f}=\left[\begin{array}{c}
-1 \\
0
\end{array}\right]
$$

and the corresponding algebraic approximation operator using Moving Least Squares function $\Phi_{i}(\boldsymbol{x})$ (shape function) formulation can be written:

$$
\mathbf{L}=\left[\begin{array}{cc}
\Phi_{x x}^{2}+\Phi_{y y}^{2} & M \Phi_{x} \\
M \Phi_{x} & \Phi_{x x}^{2}+\Phi_{y y}^{2}
\end{array}\right], \quad \mathbf{u}=\left[\begin{array}{l}
u \\
B
\end{array}\right], \quad \mathbf{f}=\left[\begin{array}{c}
-1 \\
0
\end{array}\right],
$$

for any point of the domain $\Omega$.

In Tables 1 and 2 the approximate solution of the meshless point collocation method is compared with the exact solution [30] and the numerical solution obtained with the finite element method [22] using the residual-free bubble functions, for Hartmann numbers 100 and 500, respectively, at several grid points.

In Figs. 4-6 we present velocity and magnetic field contours for $M=100,500$, or 1000 , for $\phi=\frac{\pi}{2}$ in a rectangular duct without local refinement. Results are in accordance with the corresponding in $[18,19,22]$.

Case 2: Duct with insulating walls, under the influence of an oblique magnetic field

In this case the MHD flow problem is subjected to an externally oblique magnetic field having a positive angle $\phi$ with the $y$-axis and is described from Eqs. (2.7) and (2.8). Duct has a square cross section $(|x| \leqslant 1,|y| \leqslant 1$ ) with the typical boundary
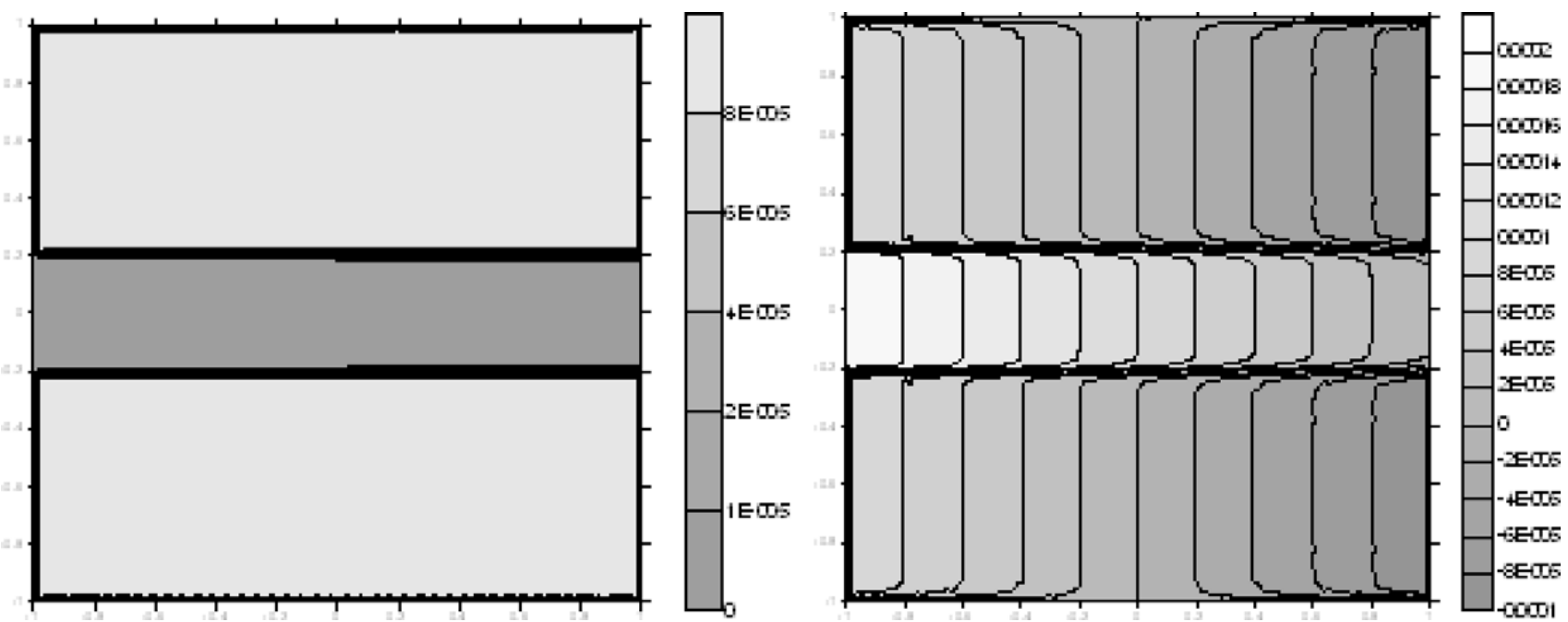

Fig. 19. Equivelocity lines and induced magnetic field lines for $M=10^{4}, \phi=\frac{\pi}{2}$ and $L=0.2$ in rectangular duct without local refinement, ( $u$, $\max =1.009132 \mathrm{E}-04, \min =0$ and $B, \max =2.00469 \mathrm{E}-04, \min =-2.00469 \mathrm{E}-04)$.
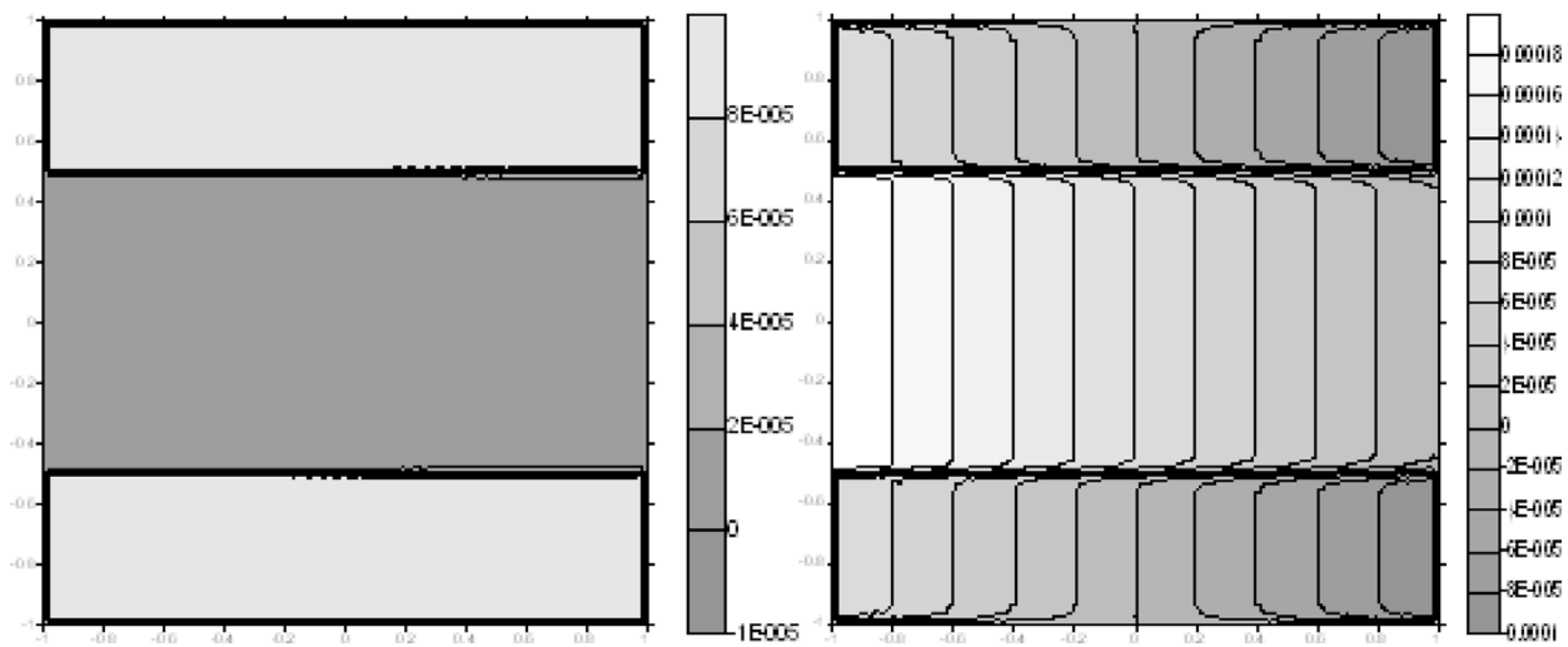

Fig. 20. Equivelocity lines and induced magnetic field lines for $M=10^{4}, \phi=\frac{\pi}{2}$ and $L=0.5$ in rectangular duct without local refinement, ( $u$, $\max =1.009132 \mathrm{E}-04, \min =0$ and $B, \max =2.00469 \mathrm{E}-04, \min =-2.00469 \mathrm{E}-04)$. 
conditions $u=B=0$ applied on the walls. Following the previous analysis, the coupled Eqs. (2.7) and (2.8) are again expressed in matrix differential operator form, $L u=f$, where

$$
\mathbf{L}=\left[\begin{array}{cc}
\nabla^{2} & M_{x} \frac{\partial}{\partial x}+M_{y} \frac{\partial}{\partial y} \\
M_{x} \frac{\partial}{\partial x}+M_{y} \frac{\partial}{\partial y} & \nabla^{2}
\end{array}\right], \quad \mathbf{u}=\left[\begin{array}{l}
u \\
B
\end{array}\right], \quad \mathbf{f}=\left[\begin{array}{c}
-1 \\
0
\end{array}\right],
$$

and, thus, in Moving Least Squares functions $\Phi_{i}(\boldsymbol{x})$ (shape function) formulation

$$
\mathbf{L}=\left[\begin{array}{cc}
\Phi_{x x}^{2}+\Phi_{y y}^{2} & M_{x} \Phi_{x}+M_{y} \Phi_{y} \\
M_{x} \Phi_{x}+M_{y} \Phi_{y} & \Phi_{x x}^{2}+\Phi_{y y}^{2}
\end{array}\right], \quad \mathbf{u}=\left[\begin{array}{l}
u \\
B
\end{array}\right], \quad \mathbf{f}=\left[\begin{array}{c}
-1 \\
0
\end{array}\right] .
$$

In Figs. 7-12 we present velocity and magnetic field contours for $M=100,500$, or 1000 and $\phi=\frac{\pi}{2}, \frac{\pi}{3}, \frac{\pi}{4}$ in a rectangular duct without local refinement. The results are in a good agreement with those obtained in [18,19].

Case 3: Duct with partly insulating, partly conducting walls

We solve the MHD equations subjected to an external magnetic field $\mathbf{B}_{0}$ in the direction of $x$-axis in a rectangular duct with a cross section $(|x| \leqslant 1,|y| \leqslant 1)$. Moreover, the rectangular duct has a conducting portion on $x=0$ line for a length $L$
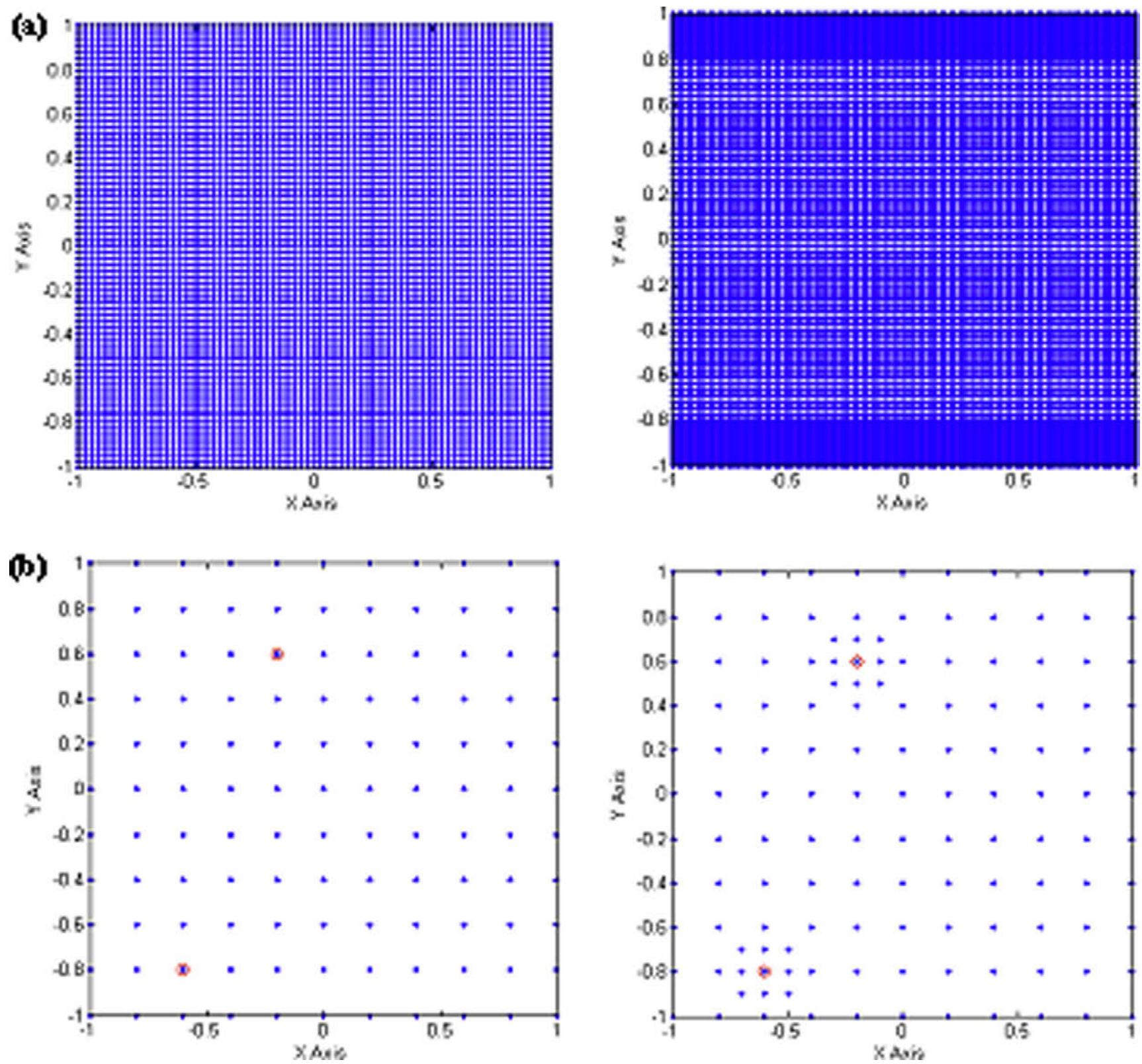

Fig. 21. (a) Nodal distribution, without refinement and with local refinement in rectangular duct when $M=10^{5}$, and (b) nodal distribution, without refinement and with local refinement in every duct for the "weak" nodes. 
symmetrically about origin. Since the applied magnetic field is in the $x$-direction, the problem is described from Eqs. (2.5) and (2.6). On the conducting portion $\frac{\partial B}{\partial \mathbf{n}}=0$ is taken.

In Figs. 13 and 14 we present velocity and magnetic field contours for $M=1000, \phi=\frac{\pi}{2}, L=0.2,0.5$, or 0.7 in a rectangular duct without local refinement. Results are in good accordance with the corresponding ones in $[18,19]$.

Case 4: Duct with partly insulating, partly conducting walls, under the influence of an oblique magnetic field

In this case the MHD flow problem is subjected to an externally oblique magnetic field having a positive angle $\varphi$ with the $y$-axis and is described from Eqs. (2.7) and (2.8). Once again, the duct has a rectangular cross section $(|x| \leqslant 1,|y| \leqslant 1)$ with the boundary conditions $u=B=0$ applied on the walls. Moreover, the rectangular duct has a conducting portion on $x=0$ line for a length $L$ symmetrically about origin. The problem is described from Eqs. (2.7) and (2.8). On the conducting portion $\frac{\partial B}{\partial n}=0$ is assumed.

In Figs. 15-18 velocity and magnetic field contours are presented for $M=1000, \phi=\frac{\pi}{3}, \frac{\pi}{4}$, and $L=0.2,0.5$, or 0.7 in a rectangular duct without local refinement.

\subsection{Large values of Hartmann number and irregular duct}

The MHD duct problem Eqs. (2.5) and (2.6) or, equivalently, Eqs. (2.7) and (2.8) can be solved in pipes with cross-sections of rectangular, circular, elliptical, or even any arbitrary type. In Figs. 19 and 20 the equivelocity lines and the induced magnetic field lines for large values of Hartmann number, $M=10^{4}$, for $\phi=\frac{\pi}{2}$ and $L=0.2$ and 0.5 in a rectangular duct are presented, without local refinement. Local refinement procedures (Fig. 21(a)) were used for Hartmann number $M=10^{5}$ and $\phi=\frac{\pi}{2}$, for a pipe with a rectangular cross-section (Fig. 22).

Indeed, an automated procedure for node refinement is proposed, based on a strong-form error mapping approach. More specifically, nodes on which the error of the calculated field property is above a user-defined threshold are extracted and surrounded by additional nodes, which are added with a predefined formulation; overall (Fig. 21(b)), an approach which ultimately converges to the solution of the governing equations with a desired accuracy. The refining method reduces the computational cost and time considerably, while leading to increasingly accurate and significantly stable results. The procedure is fully automated and robust.

Following, results are presented for circular, elliptical and arbitrary duct where $M=50$ or $200, \phi=\frac{\pi}{2}$ and $B=0$ on the boundaries. The results, where it is possible to be compared, are in very good agreement with those in $[18,19,22]$. The aforementioned geometries are irregular and, the nodal distribution inevitably can not be regular. Thus, following a procedure developed in [26], the regular nodal distribution of Type-I is embedded at the prescribed geometry, ensuring the convergence and the stability of the discrete harmonic operator. Defining the methodology for the construction of a regular grid of Type-I we address the following steps. Initially, the spatial dimensions of the geometry are defined. Following, a regular grid containing the geometry is obtained. Finally, the grid is conformed into the boundaries of the geometry (Fig. 23). Attention should be taken, such that no degenerated nodes on the boundary exist.

For the circular cross section duct, Table 3 gives a comparison between exact [31], FEM [22], BEM [23] and our MPCM results in a circular region with center at the origin, unit radius, and $M=5$. One can see that the MPCM results using 622 nodes are more accurate than the relative FEM ones by using 54 elements and 37 nodes. In Figs. 24 and 25 we present equivelocity lines and induced magnetic field lines for $M=50$ or 200, and $\phi=\frac{\pi}{2}$. Moreover, in Figs. 26 and 27 we present equivelocity lines and induced magnetic field lines for $M=50$ or 200 , and $\phi=\frac{\pi}{2}$ when the duct is elliptical.

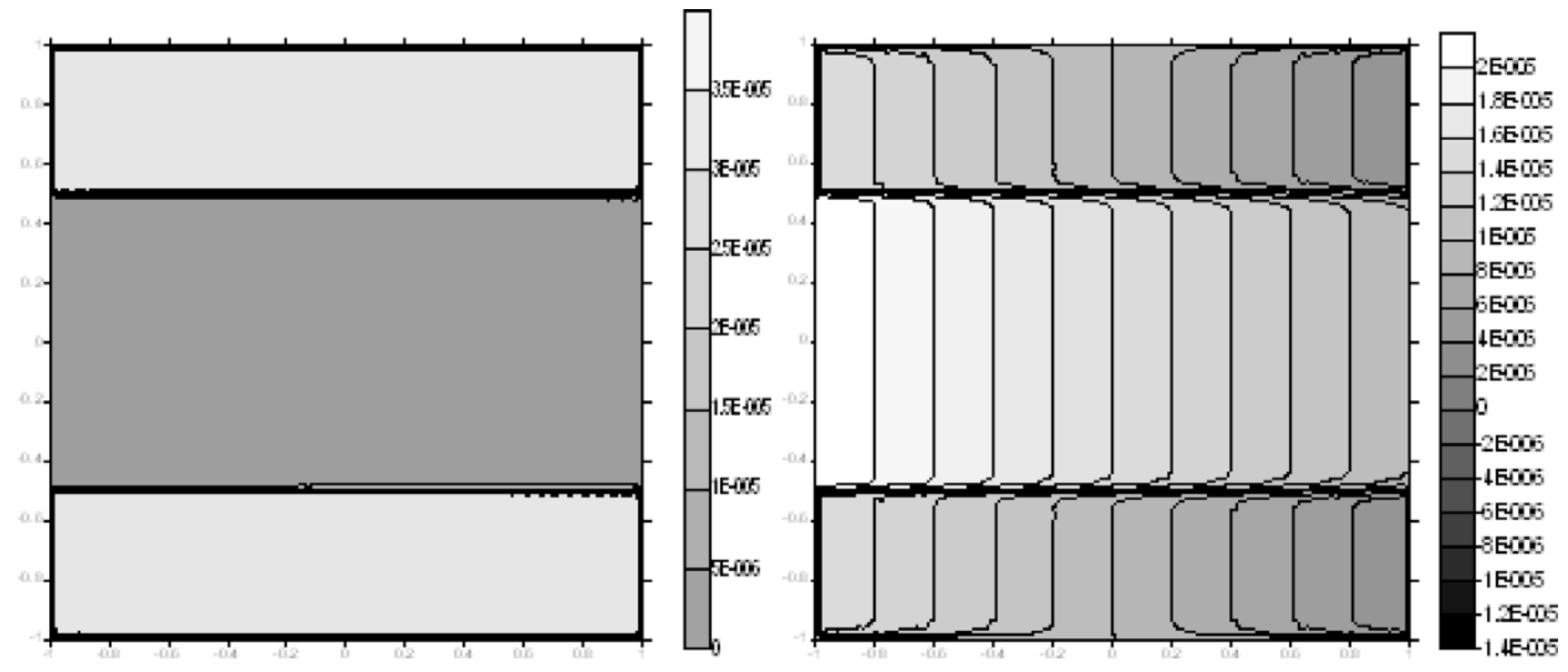

Fig. 22. Equivelocity lines and induced magnetic field lines for $M=10^{5}, \phi=\frac{\pi}{2}$ and $L=0.5$ in rectangular duct with local refinement, ( $u$, $\max =4.009132 \mathrm{E}-05, \min =0$ and $B, \max =2.00469 \mathrm{E}-05, \min =-2.00469 \mathrm{E}-05$ ). 
Additionally, a pipe flow with an arbitrary cross section is presented. The parametric curve that represents the cross section is

$$
\begin{aligned}
& x=r \cos (\theta), \\
& y=r \sin (\theta),
\end{aligned}
$$

with $r=R+g R \cos (m \theta)$ and $R=0.5, g=0.1, m=8$ and $0 \leqslant \theta \leqslant 2 \pi$.

(a)

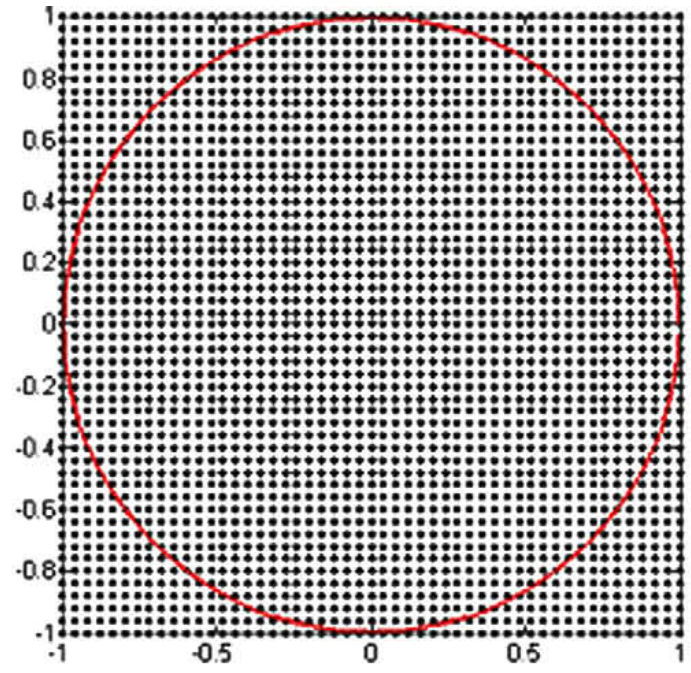

(b)

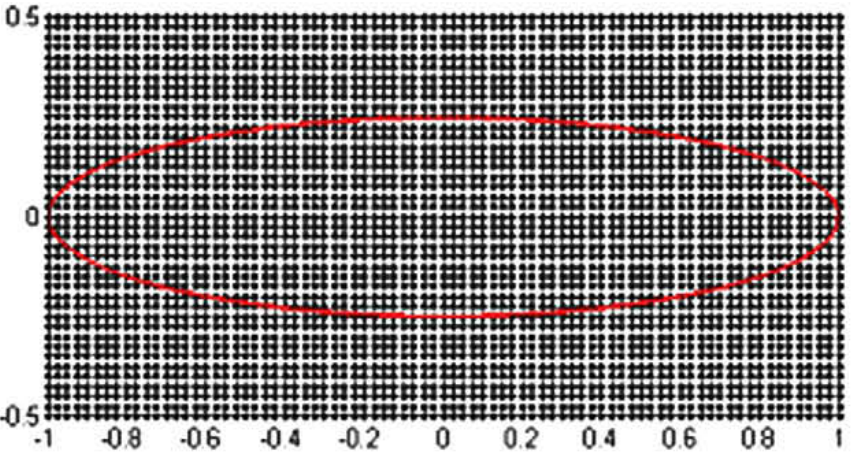

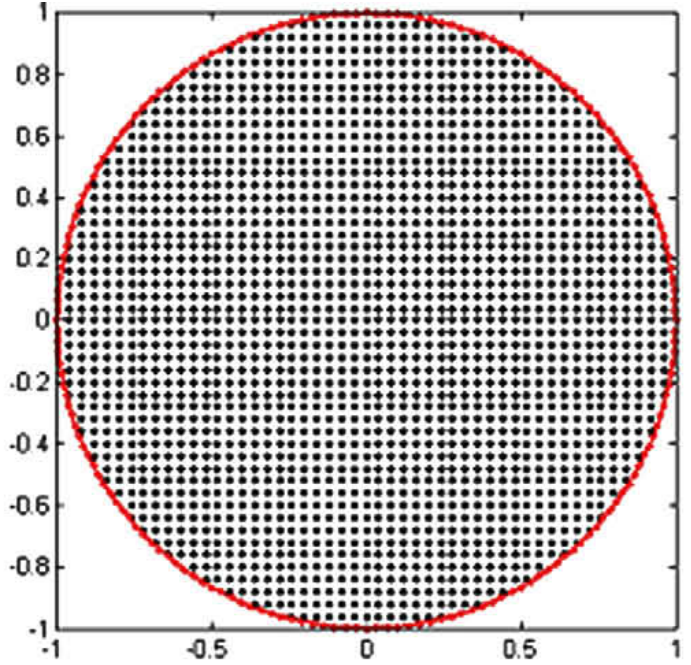

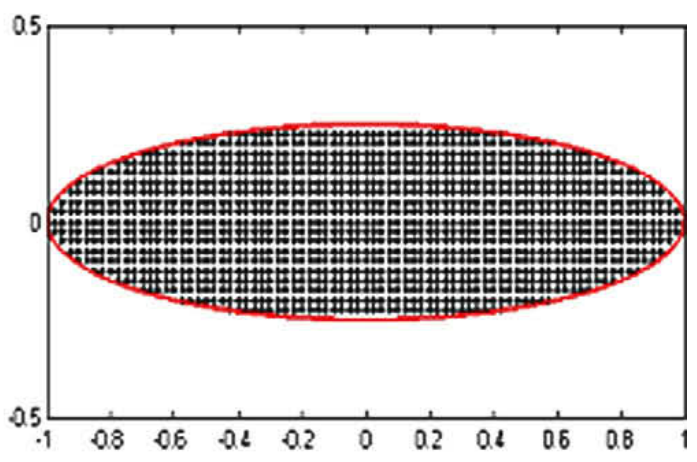

(c)
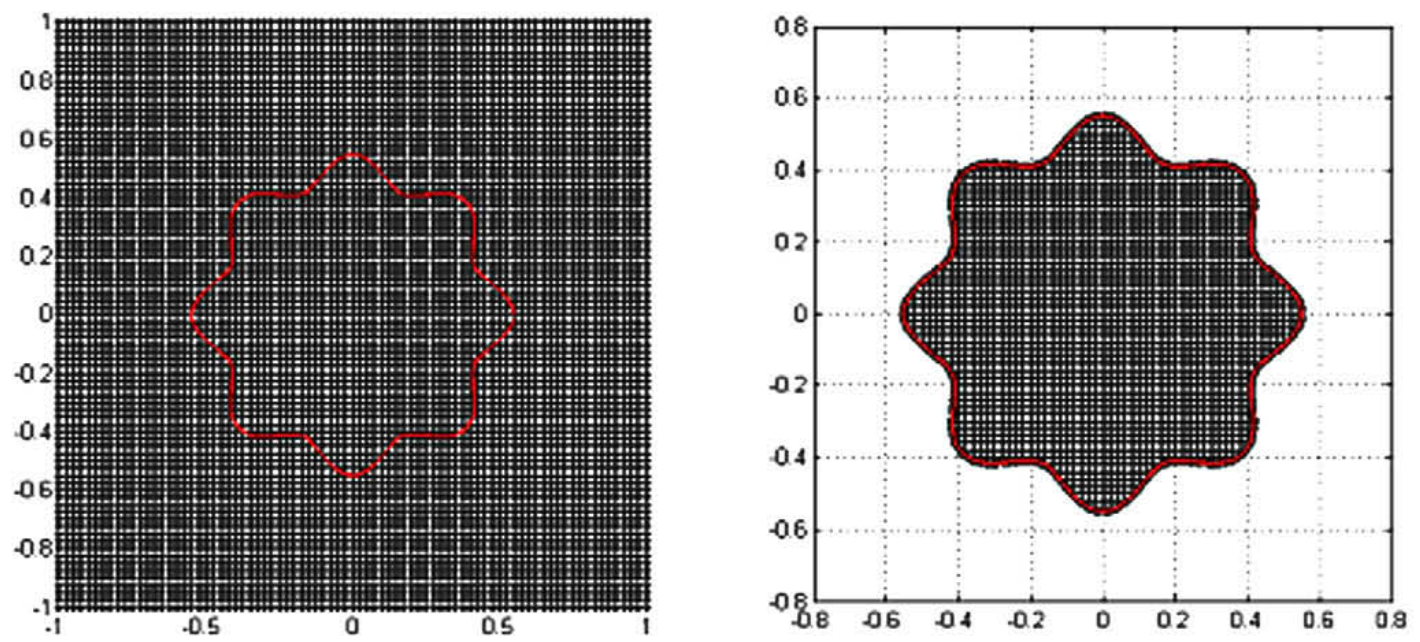

Fig. 23. Type-I nodal distribution and the final grid geometry oriented for the circular, elliptical and arbitrary cross-section duct. 
Table 3

Circular duct $(u,-B$ values for $M=5$.

\begin{tabular}{|c|c|c|c|c|c|c|c|c|}
\hline Variable & $x$ & $y$ & FEM 37n, 54e & BEM 36n, 36e & FEM 609n, 192e & BEM 300n, 300e & Exact (Gold [12]) & MPCM \\
\hline \multirow[t]{4}{*}{$u$} & 0.0 & 0.0 & 0.1571 & 0.1522 & 0.1530 & 0.1530 & 0.1530 & 0.1530 \\
\hline & $1 / 3$ & 0.0 & 0.1532 & 0.1458 & 0.1466 & 0.1466 & 0.1467 & 0.1466 \\
\hline & $2 / 3$ & 0.0 & 0.1313 & 0.1152 & 0.1165 & 0.1165 & 0.1165 & 0.1165 \\
\hline & 0.0 & $2 / 3$ & 0.0942 & 0.0904 & 0.0918 & 0.0918 & 0.0918 & 0.0918 \\
\hline \multirow[t]{4}{*}{$B$} & 0.0 & 0.0 & 0 & 0 & 0 & 0 & 0 & 0.0000 \\
\hline & $1 / 3$ & 0.0 & 0.0457 & 0.0403 & 0.0407 & 0.0407 & 0.0407 & 0.0407 \\
\hline & $2 / 3$ & 0.0 & 0.0768 & 0.0611 & 0.0624 & 0.0624 & 0.0624 & 0.0624 \\
\hline & 10 & 0.0 & 0 & 0 & 0 & 0 & 0 & 0.0000 \\
\hline
\end{tabular}
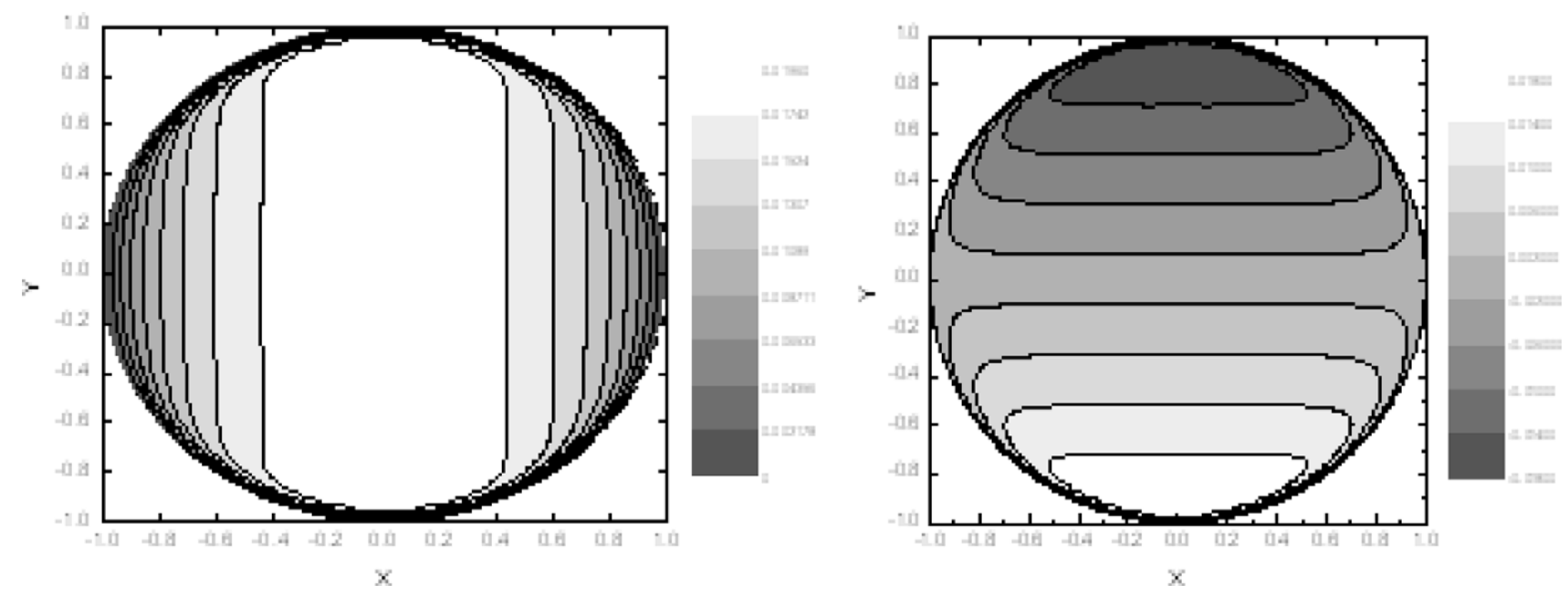

Fig. 24. Equivelocity lines and induced magnetic field lines for $M=50$ and $\phi=\frac{\pi}{2}$ in circular duct without local refinement.
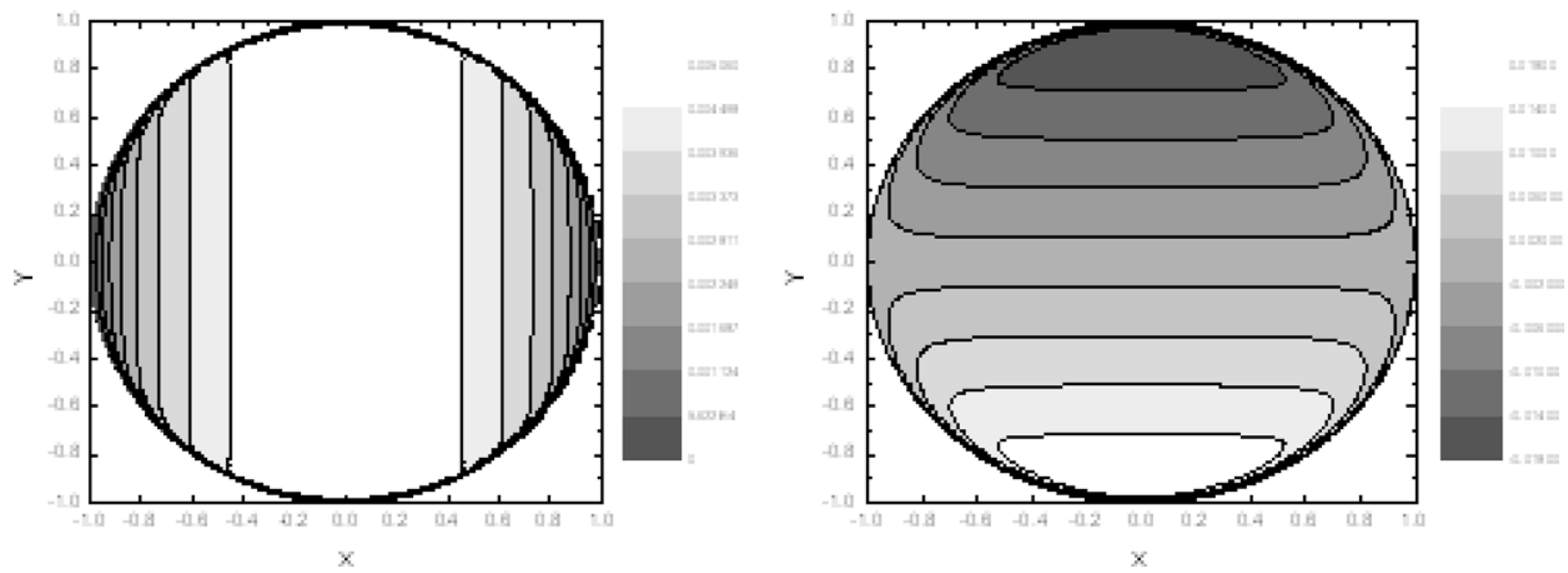

Fig. 25. Equivelocity lines and induced magnetic field lines for $M=200$ and $\phi=\frac{\pi}{2}$ in circular duct without local refinement.

As the Hartmann number $M$ increases, boundary layers are formed close to the walls of the channel. This behavior can be observed from the Figs. 28 and 29 where equivelocity lines and induced magnetic field lines are presented for $M=50$ or 200 , and $\phi=\frac{\pi}{2}$, when the duct cross section is arbitrary.

As the previous results suggest, the MPCM scheme applied here is accurate, stable and efficient, since the results are fairly close to the analytical solutions, whereas an increase of the number of nodes at specific parts of the domain (adaptation procedures) yield more accurate results, with the CPU run-time (of the code execution) remaining relatively low (Table 4).

In view of the run time of the meshless methods considered, the shape functions are not pre-defined and they must be constructed once, before the numerical solution of the resulting algebraic system. Thus, in our in-house code, the numerical procedure is primarily decomposed into two parts. Initially, the construction of the shape functions takes place, and then the solution of the resulting linear system is addressed. At the following table, Table 4, the CPU time (in seconds) for the 

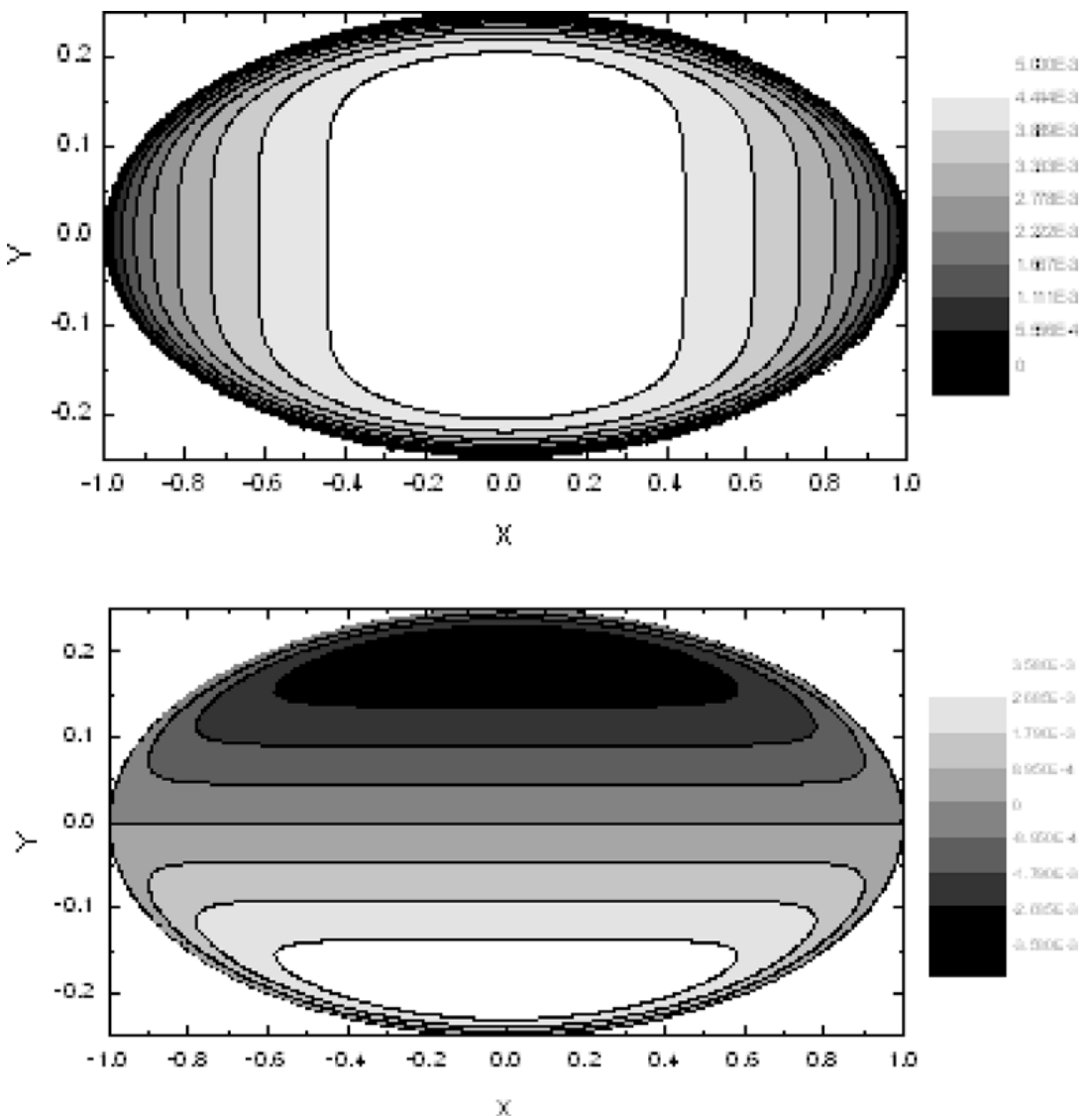

Fig. 26. Equivelocity lines and induced magnetic field lines for $M=50$ and $\phi=\frac{\pi}{2}$ in elliptical duct without local refinement.
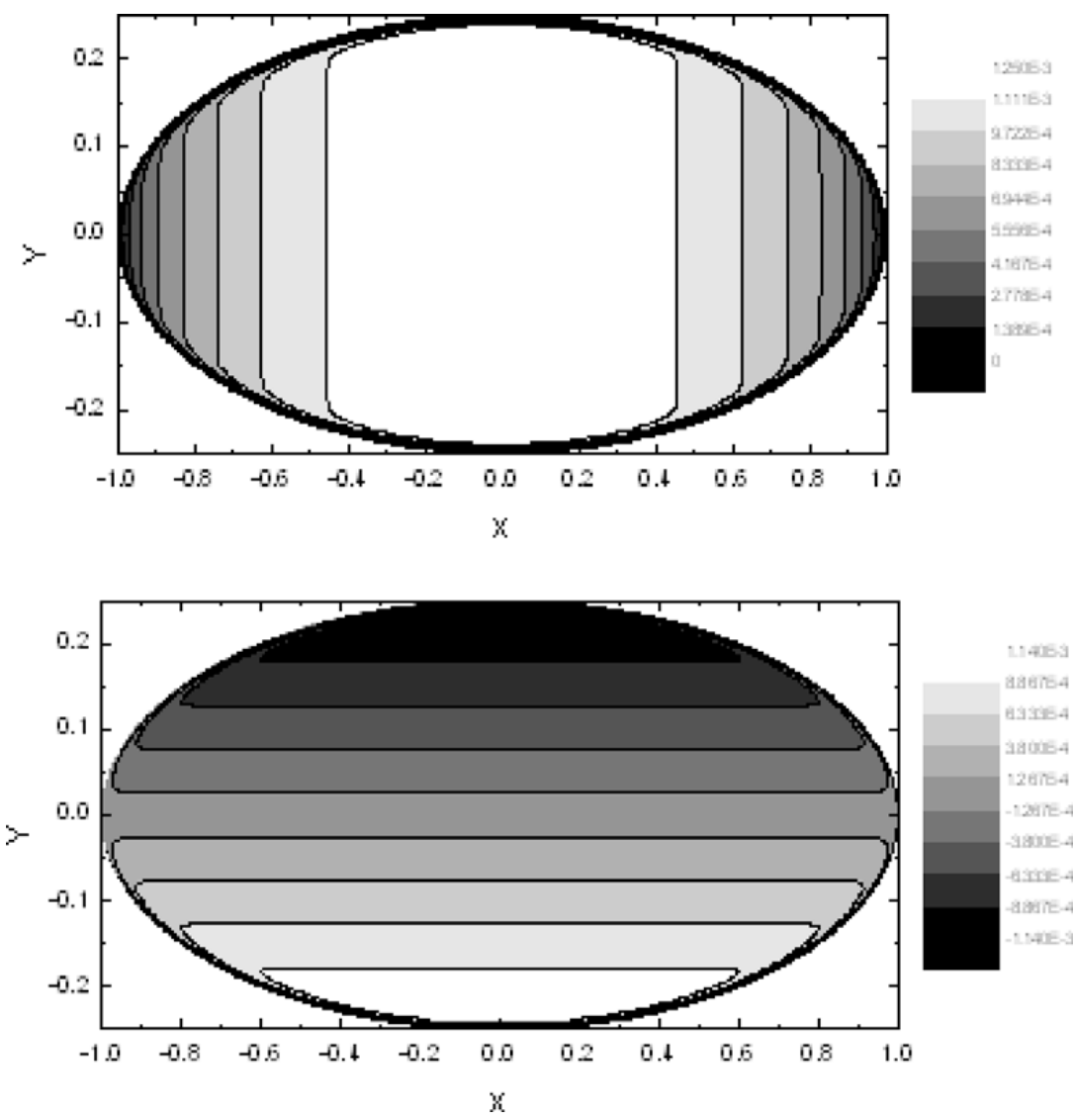

Fig. 27. Equivelocity lines and induced magnetic field lines for $M=200$ and $\phi=\frac{\pi}{2}$ in elliptical duct without local refinement. 

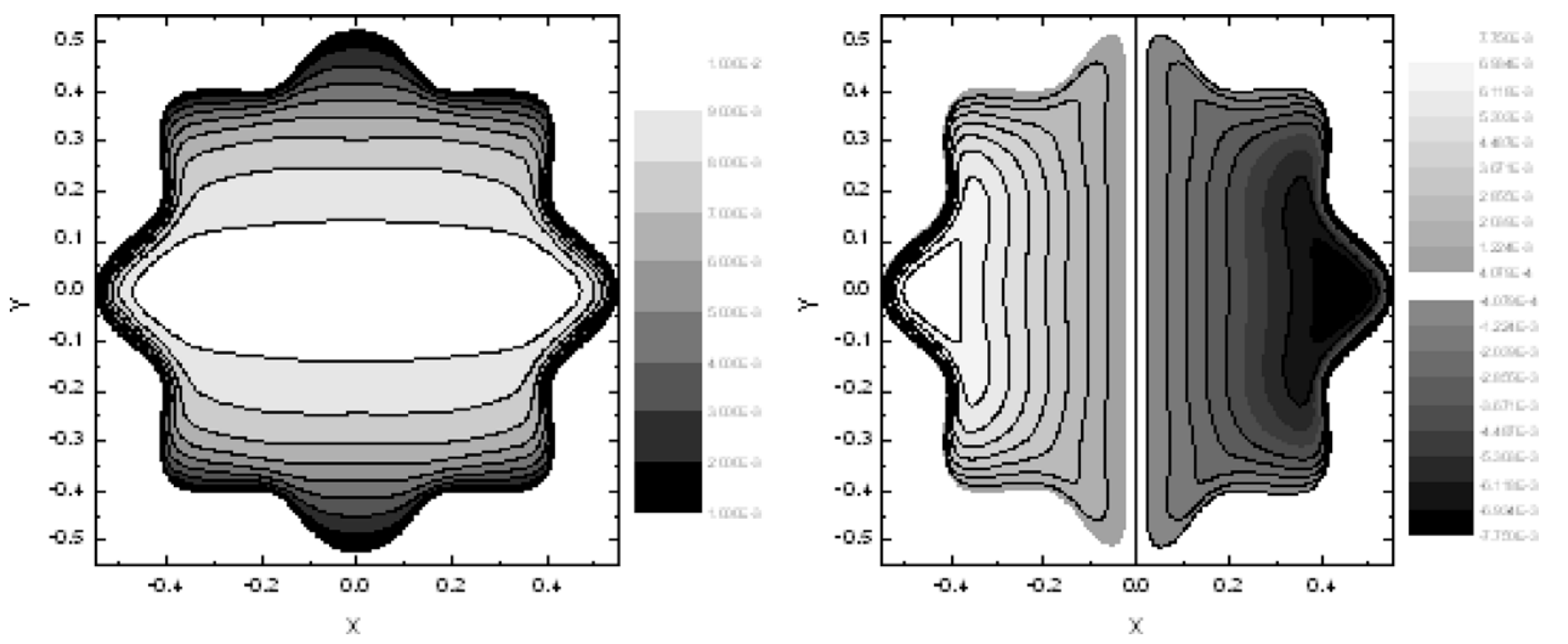

Fig. 28. Equivelocity lines and induced magnetic field lines for $M=50$ and $\phi=\frac{\pi}{2}$ in arbitrary duct without local refinement.
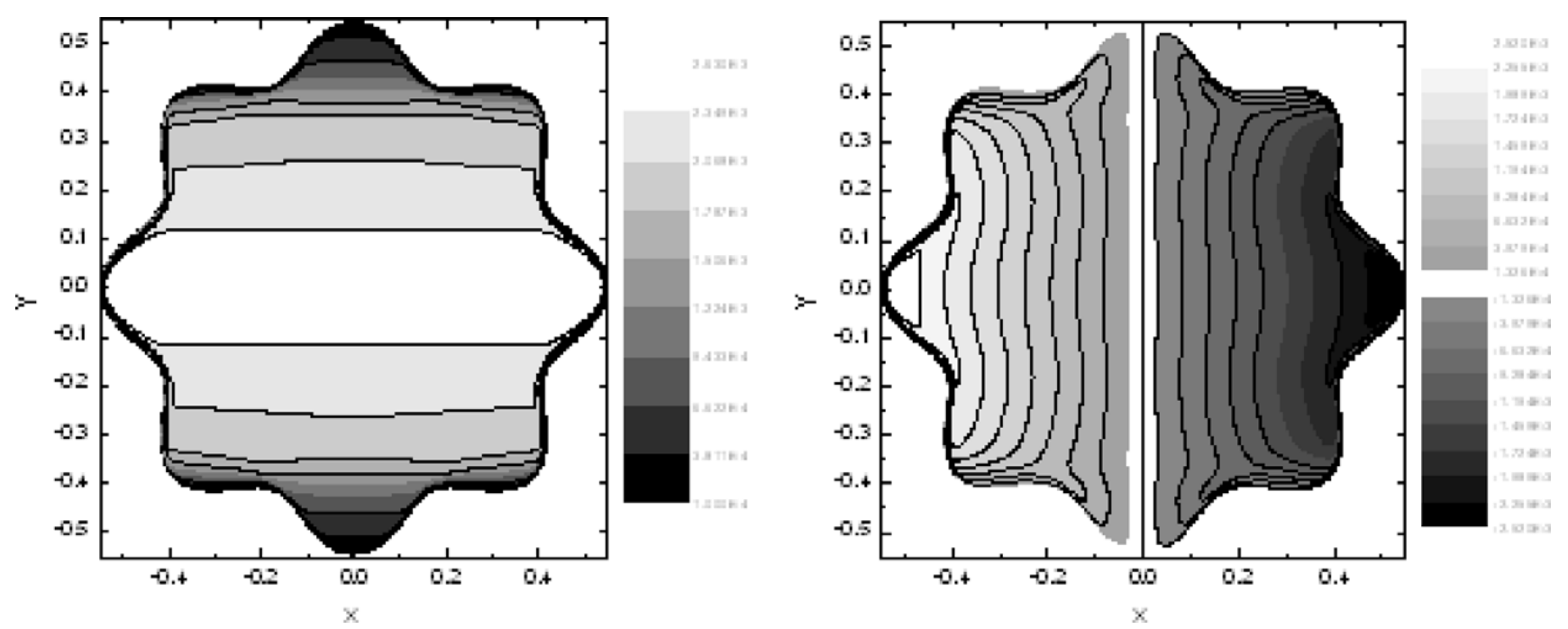

Fig. 29. Equivelocity lines and induced magnetic field lines for $M=200$ and $\phi=\frac{\pi}{2}$ in arbitrary duct without local refinement.

Table 4

CPU run-time for the MPCM scheme.

\begin{tabular}{lll}
\hline Number of nodes & Shape functions $(\mathrm{s})$ & Linear system $(\mathrm{s})$ \\
\hline 2601 & 21.17 & 1.03 \\
6561 & 60.98 & 3.03 \\
12,321 & 180.76 & 5.34 \\
30,976 & 400.46 & 51.95 \\
\hline
\end{tabular}

prescribed number of nodes is shown. The hardware characteristics used for this benchmarking are trivial, such as a CPU Pentium IV, $2.4 \mathrm{~Hz}$ with 2 GB RAM. It should be pointed out that the shape functions are only updated locally for the new nodes inserted and the surrounding nodes affected by the local support domains, thus minimizing the run-time of the shape function creation step significantly.

\section{Conclusions}

In the present study, a stable meshless point collocation method (MPCM) is developed for the solution of MHD duct problem equations with either fully insulating walls, or partially insulating and partially conducting walls. The MPCM have 
several key advantages over traditionally FEM, FDM, or BEM methods, such as the following; it requires neither domain nor surface mesh discretization, thus avoiding various topological, connectivity and dimensional difficulties of the meshing procedures; it does not involve numerical integration, while it retains a relative ease of implementation; the formulation is similar for 2D and 3D problems, and for time-dependent problems (time-varying point distributions), as well; and finally it is cost-effective due to the man-power reduction involved for the meshing steps. The main problem of these MFree techniques are some global or local stability issues at boundaries sites or internal points of increased complexity. In the present formulation, a stable MLS meshless point collocation method is used to discretize the system-governing equations. The turning point in using consistent MLS-MPCM methods which avoid the stability issues systematically is the mathematical validation of the convergence and the accuracy of their implementation on flow and diffusion problems [32]. These recently validated techniques are used here to obtain stable solutions of MHD problems. Local point refinement scheme adopted in this work uses an error indication based on the local error residuals. The adaptive procedures for additional nodes insertion around nodes of low accuracy are applied in a consistent manner. Numerical examples are given, which demonstrate the fact that the proposed adaptive meshfree method can obtain efficient and stable solutions of desired accuracy at any configuration studied. The coupled MHD equations are convection-dominated for large values of Hartmann number. Thus, the solution is obtained for values of Hartmann number up to $10^{5}$ using refined nodal distribution as $M$ increases. This significantly high value of $M$ has not been attained with previous BEM or FDM solutions. Furthermore, the numerical results presented here are obtained using second order polynomials as approximations basis, in contrast with the sophisticated bubble-functions used at the Finite Element method procedure. All the well-known characteristics of the MHD flow in ducts of arbitrary cross-sections at practically any Hartmann number can be systematically recovered with the MLS Meshless Point Collocation Method reported here.

\section{References}

[1] S. Chantasiriwan, Cartesian grid methods using radial basis functions for solving Poisson, Helmholtz, and diffusion-convection equations, Eng. Anal. Bound. Elem. 28 (2004) 1417-1425.

[2] D.L. Young, K.H. Chen, C.W. Lee, Novel meshless method for solving the potential problems with arbitrary domain, J. Comput. Phys. 209 (2005) 290321.

[3] R.A. Gingold, J.J. Monaghan, Smoothed particle hydrodynamics: theory and application to non-spherical stars, Mon. Not. Roy. Astron. Soc. 181 (1977) 275-389.

[4] B. Nayroles, G. Touzot, P. Villon, Generalizing the finite element method: diffuse approximation and diffuse elements, Comput. Mech. 10 (1992) 307318.

[5] Y.Y. Lu, T. Belytschko, L. Gu, A new implementation of the element free Galerkin method, Comput. Meth. Appl. Mech. Eng. 113 (1994) 397-414.

[6] W.K. Liu, S. Jun, Y.F. Zhang, Reproducing kernel particle methods, Int. J. Numer. Meth. Fluids 20 (1995) 1081-1106.

[7] W.K. Liu, S. Jun, S. Li, J. Adee, T. Belytschko, Reproducing kernel particle methods for structural dynamics, Int. J. Numer. Meth. Eng. 38 (1995) 16551679.

[8] J.M. Melenk, I. Babuška, The partition of unity finite element method: basic theory and applications, Comput. Meth. Appl. Mech. Eng. 139 (1996) 289314.

[9] C.A. Duarte, J.T. Oden, An h-p adaptive method using clouds, Comput. Meth. Appl. Mech. Eng. 139 (1996) $237-262$.

[10] W.K. Liu, S. Li, T. Belytschko, Moving least square reproducing kernel methods (I) methodology and convergence, Comput. Meth. Appl. Mech. Eng. 143 (1996) 422-433.

[11] T. Zhu, J. Zhang, S.N. Atluri, A meshless local boundary integral equation (LBIE) method for solving nonlinear problems, Comput. Mech. 22 (1998) 174186.

[12] S.N. Atluri, H.G. Kim, J.Y. Cho, A critical assessment of the truly meshless local Petrov-Galerkin (MLPG) methods, Comput. Mech. 24 (1999) 348-372.

[13] N.R. Aluru, A point collocation method based on reproducing kernel approximations, Int. J. Numer. Meth. Eng. 47 (2000) $1083-1121$.

[14] F. Faireeather, A. Karageorghis, The method of fundamental solutions for elliptic boundary value problems, Adv. Comput. Math. 9 (1-2) (1998) 69-95.

[15] L. Fox, P. Henrici, C.B. Moler, Approximations and bounds for eigenvalues of elliptic operators, SIAM J. Numer. Anal. 4 (1967) 89-102.

[16] P.A. Davinson, An Introduction to Magnetohydrodynamics, Cambridge University Press, 2001.

[17] E. Blums, Yu.A. Mikhailov, R. Ozols, Heat and Mass Transfer in MHD Flows, World Scientific, 1987.

[18] C.M. Tezer-Sezgin, S. Han Aydın, Solution of magnetohydrodynamic flow problems using the boundary element method, Eng. Anal. Bound. Elem. 30 (2006) 411-418.

[19] C. Bozkaya, M. Tezer-Sezgin, Fundamental solution for coupled magnetohydrodynamic flow equations, J. Comput. Appl. Math. 203 (2007) 125-144.

[20] B. Singh, J. Lal, MHD axial flow in a triangular pipe under transverse magnetic field parallel to a side of the triangle, Ind. J. Technol. 17 (1979) 184-189.

[21] B. Singh, J. Lal, Finite element method in MHD channel flow problems, Int. J. Numer. Meth. Eng. 18 (1982) $1104-1111$.

[22] A.I. Nesliturk, M. Tezer-Sezgin, The finite element method for MHD flow at high Hartmann numbers, Comput. Meth. Appl. Mech. Eng. 194 (2005) 12011224.

[23] K.E. Barrett, Duct flow with a transverse magnetic field at high Hartmann numbers, Int. J. Numer. Meth. Eng. 50 (2001) $1893-1906$.

[24] D.W. Kim, W.K. Liu, Maximum principle and convergence analysis for the meshfree point collocation method, SIAM J. Numer. Anal. 44 (2006) 515-539.

[25] G.R. Liu, Mesh Free Methods, Moving beyond the Finite Elements Method, CRC Press, 2002.

[26] G.C. Bourantas, E.D. Skouras, G.C. Nikiforidis, Adaptive support domain implementation on the moving least squares approximation for Mfree methods applied on elliptic and parabolic PDE problems using strong-form description, CMES: Comput. Model. Eng. 43 (2009) 1-25.

[27] S.N. Atluri, The Mesheless Method (MLPG) for Domain and BIE Discretizations, Tech Science Press, 2004.

[28] S.N. Atluri, H.T. Liu, Z.D. Han, Meshless local Petrov-Galerkin (MLPG) mixed collocation method for elasticity problems, CMES: Comp. Model. Eng. 14 (2006) 141-152.

[29] S.N. Atluri, H.T. Liu, Z.D. Han, Meshless local Petrov-Galerkin (MLPG) mixed finite differences method for solid mechanics, CMES: Comp. Model. Eng. 15 (2006) 1-16.

[30] J.A. Shercliff, Steady motion of conducting fluids in pipes under transverse magnetic fields, Proc. Camb. Philos. Soc. 49 (1953) $136-144$.

[31] R.R. Gold, Magnetohydrodynamic pipe flow I, J. Fluid Mech. 13 (1962) 505-512.

[32] M.G. Armentano, R.G. Durán, Error estimates for moving least square approximations, Appl. Numer. Math. 37 (2001) $397-416$. 